\title{
Warped Kähler potentials and fluxes
}

\author{
Luca Martucci \\ Dipartimento di Fisica ed Astronomia "Galileo Galilei", \\ Università di Padova \& I.N.F.N. Sezione di Padova, \\ Via Marzolo 8, 35131 Padova, Italy \\ E-mail: luca.martucci@pd.infn.it
}

ABstract: The four-dimensional effective theory for type IIB warped flux compactifications proposed in [1] is completed by taking into account the backreaction of the Kähler moduli on the three-form fluxes. The only required modification consists in a fluxdependent contribution to the chiral fields parametrising the Kähler moduli. The resulting supersymmetric effective theory satisfies the no-scale condition and consistently combines previous partial results present in the literature. Similar results hold for M-theory warped compactifications on Calabi-Yau fourfolds, whose effective field theory and Kähler potential are also discussed.

KEYwORDS: Flux compactifications, F-Theory, Superstring Vacua

ARXiv EPrint: 1610.02403 


\section{Contents}

1 Introduction 1

2 Effective theory of warped IIB models $\quad 3$

2.1 Hidden dependence of $G_{3}$ on the Kähler moduli 6

$\begin{array}{lll}2.2 & \text { Chiral coordinates } & 7\end{array}$

$\begin{array}{lll}2.3 & \text { Effective Lagrangian } & 10\end{array}$

2.4 Formulation with linear multiplets 11

3 Effective theory of warped M-theory models 13

$\begin{array}{lll}3.1 & \text { Eleven-dimensional structure } & 14\end{array}$

$\begin{array}{ll}3.2 \text { Kähler potential } & 15\end{array}$

3.3 Kähler moduli and $G_{4}$ dependence $\quad 16$

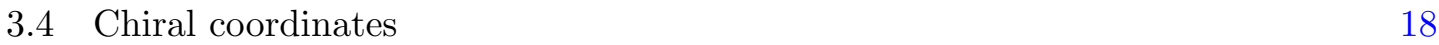

$\begin{array}{ll}3.5 & \text { Effective Lagrangian }\end{array}$

3.6 Matching with type IIB 21

4 Conclusions $\quad 22$

A IIB-theory effective theory: some details $\quad 23$

A.1 Explicit form of the warped divisor volume 23

A.2 Derivation of kinetic terms for IIB compactifications 24

B M-theory effective theory: some details $\quad 25$

B.1 Warped divisor volumes in M-theory 25

B.2 Variation of $\operatorname{Re} \rho_{a}$ in M-theory 26

B.3 Useful formulas for M-theory effective theory 27

\section{Introduction}

Type IIB/F-theory flux compactifications play a prominent role in string phenomenology, see for instance [2-4] for reviews. One important aspect of such compactifications is that fluxes and branes can backreact on the geometry generating a non-trivial warp factor, which can induce hierarchies in the energy scales perceived by the four-dimensional observers located at different points of the internal space. Nevertheless, the warp-factor is often assumed to be constant for technical simplicity, since this allows one to borrow several of the well established results on unwarped Calabi-Yau compactifications. It is then important to formulate effective four-dimensional theories that go beyond the limits of the constant warping approximation and consistently combine the effects of warping, fluxes and branes. 
In particular, the non-trivial warp factor is expected to affect the Kähler potential and the kinetic terms of the effective theory, which set the strength of physical masses and couplings, whose holomorphic structure is encoded in the F-terms. In [1], following the ideas of $[5,6]$, a derivation of the low-energy effective theory for the moduli sector of type IIB warped flux compactifications [7-9] has been proposed. ${ }^{1}$ The derivation exploits a combination of arguments based on the structure of the ten-dimensional vacua and the expected supersymmetric properties of the effective four-dimensional theory, taking advantage of its superconformal formulation at an intermediate step. Assuming that complex structure and 7-brane moduli are stabilised by fluxes, the resulting Kähler potential for the remaining massless moduli has a surprisingly simple form in terms of the universal modulus $a$ characterising this class of vacua:

$$
K=-3 \log a .
$$

The universal modulus implicitly depends on the chiral coordinates parametrising the complete set of moduli and such implicit dependence codifies the non-triviality of $K$.

The crucial step is then the determination of the appropriate complex/chiral coordinates on the moduli space. For instance, the moduli describing the positions of the mobile D3-branes inherit the complex structure of the internal complex space while the two-form potentials naturally combine into $C_{2}-\tau B_{2}$, which provides the appropriate complex parametrisation of the corresponding moduli. On the other hand, the determination of the chiral coordinates $\rho_{a}$ parametrising the Kähler and Ramond-Ramond (R-R) $C_{4}$ moduli is less obvious and in [1] they were identified by means of supersymmetric probe Euclidean D3-branes. The resulting effective Lagrangian is directly affected by the warping produced by the three-form fluxes and by the mobile D3-branes and consistently satisfies the no-scale condition $[26,27]$ in a non-obvious way.

In the derivation of [1] the three-form fluxes were considered as fixed quantities specified by certain Dolbeault cohomology classes, which stabilise the axion-dilaton and complex structure moduli and backreact on the geometry by warping it, but otherwise decouple from the low-energy dynamics. This assumption allows one to derive the effect of the threeform fluxes due to their induced D3-charge but, in fact, does not capture the complete potential impact of the fluxes on the low-energy physics. In order to understand this point, let us focus for simplicity on the vacua with constant axion-dilaton $\tau$ and recall that supersymmetry requires the three-form flux $G_{3} \equiv F_{3}-\tau H_{3}$ to define a class in the $H^{2,1}(X)$ cohomology group of the internal space $X$, which clearly does not depend on the Kähler moduli. On the other hand, $G_{3}$ is required to be primitive, that is to satisfy $J \wedge G_{3}=0$, where $J$ is the Kähler form. Such primitivity condition is trivial at the cohomological level and can be always satisfied for generic (strictly SU(3)-holonomy) compactifications, but it nevertheless introduces in $G_{3}$ a hidden dependence on the Kähler moduli. This dependence was neglected in [1] but, as we will see, its inclusion potentially affects the low-energy theory.

In this paper it is shown how such hidden dependence can be taken into account by making a minimal modification of the chiral coordinates $\rho_{a}$ proposed in [1] while keeping

\footnotetext{
${ }^{1}$ Other works on the effective theory of warped type IIB/M-theory flux compactifications include [10-25].
} 
the simple form (1.1) of the Kähler potential unchanged. This modification is sensible even if one allows for a supersymmetry breaking flux component $G^{0,3} \neq 0$ and, consistently, the Kähler potential still satisfies the no-scale condition. The resulting effective Lagrangian is almost identical to the one obtained in [1]. The only difference is in a $G_{3}$-dependent contribution to the kinetic metric for the $\rho_{a}$ moduli. Remarkably, the presence of the same contribution has already been pointed out in [22], following an approach which is somewhat orthogonal to the procedure adopted here and in [1]. Namely, the authors of [22] performed a direct and careful dimensional reduction which focuses on the $C_{4}$-moduli and keeps the (non-universal) Kähler moduli fixed - see also the very recent [25] for a similar derivation including mobile D3-branes. Hence, the results of the present paper provide a manifestly supersymmetric completion of the results of $[22,25]$ and resolve the apparent conflict between the approaches adopted in $[22,25]$ and in $[1,5,6]$.

In section 2 , the above-mentioned $G_{3}$-induced modifications of the effective theory are derived and discussed for the subclass of type IIB compactifications on warped CalabiYau spaces with constant axion-dilaton and no seven-branes. A purely type IIB extension of the results to F-theory models, although in principle straightforward, is complicated by the non-trivial transformation properties of $G_{3}$ under the $\operatorname{SL}(2, \mathbb{Z})$ duality group and by its entanglement with the fluxes supported on 7-branes. On the other hand, the Ftheory generalisation of the results of section 2 admit a simple formulation in the dual M-theory framework [28], in which the type IIB bulk and seven-brane fluxes are unified in the background $G_{4}$-flux. This is shown in section 3, which considers M-theory warped flux compactifications to three-dimensions on general (non-necessarily elliptically fibered) Calabi-Yau four-folds $[29,30]$. In section 3 we show how the logic followed in [1] and in section 2 of the present paper can be readily adapted to the M-theory framework, leading to a simple Kähler potential similar to (1.1) and to chiral coordinates that allow for the incorporation of warping, mobile M2-branes and fluxes into the effective theory. In particular, we will see that the resulting effective Lagrangian contains manifestly $G_{4}$ dependent terms, analogous to the type IIB $G_{3}$ dependent terms discussed above.

\section{Effective theory of warped IIB models}

Let us review the structure of IIB/F-theory warped flux compactifications [7-9], mostly following the notation of [1]. These vacua have an Einstein frame metric of the form

$$
\mathrm{d} s_{10}^{2}=\ell_{\mathrm{S}}^{2} e^{2 A}|\Psi|^{2} \mathrm{~d} s_{4}^{2}+\ell_{\mathrm{S}}^{2} e^{-2 A} \mathrm{~d} s_{X}^{2}
$$

where $\ell_{s} \equiv 2 \pi \sqrt{\alpha^{\prime}}$ is the string length scale, $\mathrm{d} s_{4}^{2}$ is the external four-dimensional Minkowski metric, $\mathrm{d} s_{X}^{2}$ is the metric of the internal Kähler space $X$ and $e^{A}$ represents the warp factor which varies along the internal directions $y^{m}, m=1, \ldots, 6 . \Psi$ is the conformal compensator, which has dimension of a mass and is eventually fixed in order to obtain a canonically normalised four-dimensional supergravity in the Einstein frame. Note that we have introduced the $\ell_{\mathrm{s}}^{2}$ factor in order to work with natural units along the internal space. 
The warp factor is sourced by the D3-charges present in the internal space and determined by the equation

$$
\Delta_{X} e^{-4 A}=\frac{1}{\ell_{\mathrm{s}}^{4}} *_{X} Q_{6}
$$

with $Q_{6}$ representing the D3-charge density

$$
Q_{6}=\ell_{\mathrm{s}}^{4} \sum_{I \in \mathrm{D} 3 \text { 's }} \delta_{I}^{6}-\frac{\mathrm{i}}{2 \operatorname{Im} \tau} G_{3} \wedge \bar{G}_{3}+Q_{6}^{\mathrm{nd}}
$$

where $\delta_{I}^{6}$ are delta-like 6-forms associated with the mobile D3-branes, while $Q_{6}^{\text {nd }}$ is some additional contribution to the D3-charge, for instance due to O3-planes or curvature corrections on 7-branes, which we assume non-dynamical. The internal part of the self-dual five-form flux $F_{5}$ is completely fixed in terms of the warping, $F_{5}^{\text {int }}=\ell_{\mathrm{s}}^{4} *_{X} \mathrm{~d} e^{-4 A}$, and the associated tadpole condition reads

$$
\int_{X} Q_{6}=0
$$

The general solution of $(2.2)$ can be written in the form

$$
e^{-4 A}=a+e^{-4 A_{0}}
$$

where $a$ is the universal modulus and $e^{-4 A_{0}(y)}$ is the particular solution of (2.2) fixed by the condition

$$
\int_{X} e^{-4 A_{0}} \operatorname{dvol}_{X}=0
$$

Notice that $e^{-4 A_{0}(y)}$ can also assume negative values very close to sources with negative tension, where the supergravity approximation breaks down.

The internal Kähler metric $\mathrm{d} s_{X}^{2}$ has fixed (dimensionless) volume $\mathrm{v}_{0}$ :

$$
\int_{X} \mathrm{dvol}_{X}=\frac{1}{3 !} \int_{X} J \wedge J \wedge J=\mathrm{v}_{0}
$$

where $J$ is the Kähler form. ${ }^{2}$ This does not imply a loss of any degree of freedom, since the overall breathing mode is parametrised by the universal modulus $a$. The remaining nonuniversal Kähler moduli $v^{a}, a=1, \ldots, h^{1,1}(X)-1$, can be identified with the coefficients of the expansion

$$
J=v^{a} \omega_{a}
$$

where $\omega_{a}$ are integral harmonic $(1,1)$-forms which provide a basis of $H^{2}(X ; \mathbb{Z})$. Because of (2.7), these non-universal Kähler moduli satisfy the constraint

$$
\frac{1}{3 !} \mathcal{I}_{a b c} v^{a} v^{b} v^{c}=\mathrm{v}_{0}
$$

where we have introduced the intersection numbers

$$
\mathcal{I}_{a b c} \equiv \int_{X} \omega_{a} \wedge \omega_{b} \wedge \omega_{c} .
$$

\footnotetext{
${ }^{2}$ The notation of [1] has been slightly simplified. In particular $a, e^{-4 A_{0}}, \mathrm{~d} s_{X}^{2}$ and $J$ here correspond to $\hat{a}, e^{-4 \hat{A}_{0}}, \mathrm{~d} s_{X, 0}^{2}$ and $J_{0}$ there.
} 
In the following a key role is played by the three-form flux

$$
G_{3} \equiv F_{3}-\tau H_{3}
$$

which, away from fluxed 7-branes, must satisfy the Bianchi identities $\mathrm{d} F_{3}=\mathrm{d} H_{3}=0$. In order to solve the equations of motion, $G_{3}$ must be imaginary-self-dual $\left({ }_{X} G_{3}=\mathrm{i} G_{3}\right)$ that is, it must have vanishing components $G^{1,2}=G^{3,0}=0$ and be primitive:

$$
J \wedge G_{3}=0
$$

This condition is accompanied by an analogous primitivity condition on the 7-branes fluxes. Furthermore, the $(2,1)$ and primitive component $G_{\mathrm{P}}^{2,1}$ preserves four-dimensional $\mathcal{N}=1$ supersymmetry, while a non vanishing $(0,3)$ component $G^{0,3}$ breaks supersymmetry in a (classically) controlled way.

The background fluxes generically stabilise complex structure and seven-brane moduli, which can then be assumed to decouple at low energy. In such a case, in [1] it was argued that the (implicitly defined) Kähler potential describing the moduli sector of the fourdimensional effective theory has the following simple form

$$
K=-3 \log a
$$

up to an additional constant which depends on the specific normalisation of the flux superpotential and of the conformal compensator.

Let us briefly recall the argument leading to (2.13). The metric ansatz (2.1) is invariant under a simultaneous constant rescaling of the four-dimensional metric and the compensator: $\mathrm{d} s_{4}^{2} \rightarrow e^{-2 \omega} \mathrm{d} s_{4}^{2}, \Psi \rightarrow e^{\omega} \Psi$. This rigid redundancy is promoted to a local four-dimensional one once the four-dimensional fields are allowed to have a non-trivial lowenergy dynamics. Hence the effective four-dimensional theory is expected to be invariant under arbitrary Weyl transformations. This property, as well as its supersymmetric generalisation $[1,5,6]$, is accommodated by the superconformal formulation of four-dimensional supergravity, see for instance [31] for a detailed discussion and references to the original papers. Such a formulation has a non-canonical Einstein-Hilbert term

$$
\frac{1}{2} C \int \sqrt{-g_{4}}|\Psi|^{2} e^{-\frac{1}{3} K} R_{4}
$$

where $C$ is some arbitrary positive constant and $K$ is the Kähler potential of the ordinary four-dimensional supergravity, with canonical Einstein-Hilbert term $\frac{1}{2} M_{\mathrm{P}}^{2} \int \sqrt{-g_{4}} R_{4}$, that is obtained by gauge-fixing the superconformal symmetry [31]. Clearly $C$ may be reabsorbed in a rescaling of $\Psi$ and can be chosen in order to simplify the relation between the ten- and four-dimensional quantities.

By matching (2.14) with the ten-dimensional term $\frac{2 \pi}{\ell_{\mathrm{s}}^{8}} \int \sqrt{-g_{10}} R_{10}$ evaluated on (2.1), one readily identifies

$$
C e^{-\frac{1}{3} K}=4 \pi \int_{X} e^{-4 A} \mathrm{dvol}_{X}=4 \pi \mathrm{v}_{0} a
$$

where we have used (2.5) and (2.6). We can then choose $C=4 \pi \mathrm{v}_{0}$ and arrive at (2.13). ${ }^{3}$ With this choice, the gauge-fixing to ordinary supergravity is achieved by setting $\Psi=$ $\frac{M_{\mathrm{P}}}{\sqrt{4 \pi \mathrm{v}_{0}}} e^{\frac{1}{6} K}=\frac{M_{\mathrm{P}}}{\sqrt{4 \pi \mathrm{v}_{0} a}}$.

\footnotetext{
${ }^{3}$ Note that $C=1$ in [1] and then the Kähler potentials here and there differ by a shift of $3 \log \left(4 \pi \mathrm{v}_{0}\right)$.
} 


\subsection{Hidden dependence of $G_{3}$ on the Kähler moduli}

In order to make our point as clean as possible, we now focus on warped Calabi-Yau compactifications, hence excluding 7-branes while retaining mobile D3-branes, O3-planes and a non-trivial three-form flux $G_{3} \neq 0$. In this case the axion-dilaton $\tau$ is constant and the internal space can be written as $X=\hat{X} / \mathbb{Z}_{2}$, where $\hat{X}$ is a Calabi-Yau space and $\mathbb{Z}_{2}$ represents the orientifold involution. In particular, the different fields on $X$ uplift to fields on $\hat{X}$ of appropriate parity under the orientifold involution. For instance, the Kähler form $J$ is even while $G_{3}$ is odd, and the harmonic forms $\omega_{a}$ provide a basis of the integral even cohomology group $H_{+}^{2}(X ; \mathbb{Z})$. Since $\tau$ is constant, $G_{3}=G^{2,1}+G^{0,3}$ is actually harmonic and then defines an element of the odd cohomology groups $H_{-}^{2,1}(X) \oplus H_{-}^{0,3}(X)$. CalabiYau three-folds have vanishing Hodge numbers $h^{1,0}=h^{3,2}=0$. Hence the right-hand side of (2.12) is always satisfied in cohomology and does not lead to any constraint on the Kähler moduli.

On the other hand, the condition (2.12) introduces in $G_{3}$ a hidden dependence on the (non-universal) Kähler moduli $v^{a}$ defined in (2.8). Indeed, let us consider an infinitesimal variation $\delta v^{a}$, preserving the constraint (2.9). Being the cohomology class of $G_{3}$ fixed, we can write the corresponding variation as $\delta G_{3}=\mathrm{d} \delta \mathcal{B}_{2}$, with $\delta \mathcal{B}_{2}=\delta C_{2}-\tau \delta B_{2}$. Since $\delta J=\delta v^{a} \omega_{a}$, the preservation of (2.12) under Kähler moduli deformations requires

$$
\delta v^{a} \omega_{a} \wedge G_{3}+J \wedge \delta G_{3}=0 .
$$

Observing that the possible supersymmetry-breaking $(0,3)$-component $G^{0,3}$ is automatically primitive and then is not affected by the variation of the Kähler moduli, we can impose $\delta G^{3,0}=\delta G^{1,2}=\delta G^{0,3}=0$, which allow us to restrict $\delta \mathcal{B}_{2}$ to be $(1,1)$ and $\bar{\partial}$-closed. We can then expand $\delta \mathcal{B}_{2}=\delta \mathcal{B}_{\mathrm{h}}^{1,1}+\bar{\partial} \delta \Lambda^{1,0}$, where $\delta \mathcal{B}_{\mathrm{h}}^{1,1}$ is harmonic and describes a variation of the $\left(B_{2}, C_{2}\right)$ moduli, while $\delta \Lambda^{1,0}$ is a complex $(1,0)$-form. Note that the one-form $\delta \Lambda^{1,0}$ is determined up to a $\partial$-exact piece. We can then expand

$$
\delta \Lambda^{1,0}=\delta v^{a} \Lambda_{a}^{1,0}
$$

with $\Lambda_{a}^{1,0}$ such that

$$
v^{a} \Lambda_{a}^{1,0}=0
$$

up to a $\partial$-exact contribution. The condition (2.18) ensures that $\delta v^{a} \Lambda_{a}^{1,0}$ is trivial for $\delta v^{a}$ proportional to $v^{a}$, i.e. for deformations orthogonal to the constraint (2.9). Hence we can set

$$
\delta G_{3}=\delta v^{a} \partial \bar{\partial} \Lambda_{a}^{1,0}
$$

and this in turn implies that (2.16) can be rewritten as

$$
J \wedge \partial \bar{\partial} \Lambda_{a}^{1,0}=-\omega_{a} \wedge G_{3} .
$$

Note that, consistently, both sides of this equation vanish once contracted with $v^{a}$.

We may now remove the arbitrary $\partial$-exact contribution to $\Lambda_{a}^{1,0}$ by imposing $\partial^{\dagger} \Lambda_{a}^{1,0}=0$ and rewrite (2.20) as

$$
\Delta_{X} \Lambda_{a}^{1,0}=-2 *_{X}\left(\omega_{a} \wedge G_{3}\right)
$$


where $\Delta_{X} \equiv \mathrm{dd}^{\dagger}+\mathrm{d}^{\dagger} \mathrm{d} \equiv 2\left(\bar{\partial}^{\dagger} \bar{\partial}+\bar{\partial} \bar{\partial}^{\dagger}\right)$ is the usual Laplacian. ${ }^{4}$ Hodge theorem then implies that $\Lambda_{a}^{1,0}$ is uniquely determined in terms of $\omega_{a} \wedge G_{3}$ (up to a $\partial$-exact term, if one removes the gauge-fixing condition $\partial^{\dagger} \Lambda_{a}^{1,0}=0$ ). From the primitivity condition (2.12) it also follows that (2.21) actually requires (2.18), showing the mutual consistency of these equations.

Equation (2.19) specifies the way in which the $G_{3}$ flux carries the hidden dependence on the Kähler moduli. Hence, we can split $G_{3}$ into the sum of a fixed moduli-independent contribution $G_{3}^{(0)}$, in the same $H^{2,1}(X) \oplus H^{0,3}(X)$ cohomology class of $G_{3}$, plus a moduli dependent $(2,1) \bar{\partial}$-exact piece. If we locally set $G_{3}^{(0)}=\mathrm{d} \mathcal{B}_{2}^{(0)}$, by repeating the same argument followed above we can locally write $G_{3}=\mathrm{d} \mathcal{B}_{2}$ with

$$
\mathcal{B}_{2}=\mathcal{B}_{2}^{(0)}+\bar{\partial} b^{1,0}+\ell_{\mathrm{s}}^{2} \beta^{\alpha} \chi_{\alpha}
$$

where $b^{1,0}$ is some globally defined $(1,0)$-form and $\chi_{\alpha}, \alpha=1, \ldots, h_{-}^{1,1}$, are odd harmonic $(1,1)$-forms defining a basis of $H_{-}^{2}(X ; \mathbb{Z})$. The coefficients $\beta^{\alpha}$ give the complex parametrisation of the $\left(B_{2}, C_{2}\right)$-moduli and will enter the four-dimensional effective theory as chiral fields. On the other hand, the one-form $b^{1,0}$ carries the non-trivial dependence of $G_{3}$ on the non-universal Kähler moduli. More explicitly, we can write

$$
G_{3}=G_{3}^{(0)}+\partial \bar{\partial} b^{1,0}
$$

with

$$
\delta b^{1,0}=\delta v^{a} \Lambda_{a}^{1,0} .
$$

\subsection{Chiral coordinates}

We are now in the position to revisit the identification of the moduli chiral coordinates proposed in [1]. As we have already observed, the $\left(B_{2}, C_{2}\right)$ moduli are naturally parametrised by the complex coordinates $\beta^{\alpha}$, while the D3-brane moduli are described by a set of coordinates $Z_{I}^{i}$, with $I=1, \ldots, N_{\mathrm{D} 3}$, which specify the positions of the mobile D3-branes in some local complex coordinate system $z^{i}$ along $X$.

On the other hand, the identification of a set of chiral coordinates $\rho_{a}, a=1, \ldots, h_{+}^{1,1}$, parametrising the universal modulus $a$, the non-universal Kähler moduli $v^{a}$ and the $C_{4^{-}}$ moduli is less obvious. In order to detect them, [1] used an argument based on supersymmetric probe D3-brane instantons, working under the assumption that $G_{3}$ is decoupled from the low-energy dynamics. As we have shown in section 2.1, such an assumption misses a hidden $G_{3}$ dependence on the Kähler moduli. Let us then revisit the procedure followed in [1], now taking into account this additional ingredient.

Consider a Euclidean supersymmetric D3-brane, E3-brane for short, wrapping an effective (even) divisor $D$ and supporting an anti-self-dual world-volume flux $\mathcal{F}=\frac{1}{2 \pi} \ell_{\mathrm{s}}^{2} F_{\mathrm{D} 3}-$ $\left.B_{2}\right|_{D}$. Its on-shell action is complex and must depend holomorphically on the background chiral moduli. Having the possibility to choose $h_{+}^{1,1}$ independent (even) divisors

\footnotetext{
${ }^{4}$ Note that $\Lambda_{a}^{1,0}$ can be identified with a corresponding one-form appearing in [22, 25], see for instance eq. (3.39) in [25], where the condition (2.21) arises as a constraint for a dimensional reduction performed at fixed non-universal Kähler moduli. See later for more comments on this point.
} 
$D_{a}, a=1, \ldots, h_{+}^{1,1}$, there are enough probe E3-branes for identifying a set of chiral coordinates $\rho_{a}$, which should enter as an integral linear combination the on-shell action of any supersymmetric E3-brane. In fact, for our purposes, we can focus on $\operatorname{Re} \rho_{a}$ and then consider just the real DBI contribution to the E3-brane action. Indeed, the imaginary part of $\rho_{a}$ depends just on the R-R axionic moduli, which do not appear in (2.13) and must correspond to (perturbative) isometries of the theory.

Supersymmetry of the E3-brane is equivalent to a generalised calibration condition [5, 32], which implies that the on-shell bosonic DBI action takes the form

$$
\frac{1}{2 \pi} S_{\mathrm{DBI}}=\frac{1}{2} \int_{D} e^{-4 A} J \wedge J-\frac{1}{2 \ell_{\mathrm{s}}^{4}} \operatorname{Im} \tau \int_{D} \mathcal{F} \wedge \mathcal{F}
$$

We can choose a basis of even divisors $D_{a}$ which are Poicaré dual to the harmonic forms $\omega_{a}$ and decompose $D=n^{a} D_{a}$. Then, we would like to identify $\operatorname{Re} \rho_{a}$ such that

$$
\frac{1}{2 \pi} S_{\mathrm{DBI}}=n^{a} \operatorname{Re} \rho_{a}+(\mathrm{hol}+\overline{\mathrm{hol}})
$$

where $($ hol $+\overline{\text { hol }})$ denotes the real part of a holomorphic function of $\left(Z_{I}^{i}, \beta^{\alpha}\right)$. As in [1], by extracting the dependence of $S_{\mathrm{DBI}}$ on the background moduli we will arrive at our definition of $\operatorname{Re} \rho_{a}$. The new contribution arising from the hidden Kähler moduli dependence discussed in section 2.1 affects only the second term on the right hand-side of (2.25), so we can focus on that.

By using (2.22) we can decompose $\mathcal{F}$ as follows

$$
\mathcal{F}=\mathcal{F}^{(0)}+\left.\frac{1}{\operatorname{Im} \tau}\left[\operatorname{Im}\left(\bar{\partial} b^{1,0}\right)+\ell_{\mathrm{s}}^{2} \operatorname{Im} \beta^{\alpha} \chi_{\alpha}\right]\right|_{D}
$$

and then, by performing some integrations by parts, we can write

$$
\begin{aligned}
\frac{1}{2 \ell_{\mathrm{s}}^{4}} \operatorname{Im} \tau \int_{D} \mathcal{F} \wedge \mathcal{F}= & \frac{1}{2 \operatorname{Im} \tau} \operatorname{Im} \beta^{\alpha} \operatorname{Im} \beta^{\beta} \int_{D} \chi_{\alpha} \wedge \chi_{\beta} \\
& +\frac{1}{2 \operatorname{Im} \tau \ell_{\mathrm{s}}^{4}} \int_{D}\left[\operatorname{Re}\left(b^{1,0} \wedge \bar{G}_{3}\right)-\frac{1}{2} \bar{\partial} b^{1,0} \wedge \partial \bar{b}^{0,1}\right] \\
& +\frac{1}{\ell_{\mathrm{s}}^{2}} \operatorname{Im} \beta^{\alpha} \int_{D} \mathcal{F}^{(0)} \wedge \chi_{\alpha}+\frac{1}{2 \ell_{\mathrm{s}}^{4}} \operatorname{Im} \tau \int_{D} \mathcal{F}^{(0)} \wedge \mathcal{F}^{(0)}
\end{aligned}
$$

The last line can be considered as contributing to the $($ hol $+\overline{\text { hol }})$ part in $(2.26)$ and can then be discarded. On the other hand, as shown in [1] and reviewed in appendix A.1, the dependence of the first term in (2.25) on the background moduli can be made explicit, by writing $\frac{1}{2} \int_{D_{a}} e^{-4 A} J \wedge J$ as in (A.8).

By combining these results, our guiding condition (2.26) leads to the following definition of the real part of the chiral coordinates $\rho_{a}$ :

$$
\begin{aligned}
\operatorname{Re} \rho_{a} \equiv & \frac{1}{2} a \mathcal{I}_{a b c} v^{b} v^{c}+\frac{1}{2} \sum_{I} \kappa_{a}\left(Z_{I}, \bar{Z}_{I} ; v\right)+h_{a}(v)-\frac{1}{2 \operatorname{Im} \tau} \mathcal{I}_{a \alpha \beta} \operatorname{Im} \beta^{\alpha} \operatorname{Im} \beta^{\beta} \\
& -\frac{1}{2 \operatorname{Im} \tau \ell_{\mathrm{S}}^{4}} \int_{D_{a}}\left[\operatorname{Re}\left(b^{1,0} \wedge \bar{G}_{3}\right)-\frac{1}{2} \bar{\partial} b^{1,0} \wedge \partial \bar{b}^{0,1}\right] .
\end{aligned}
$$


In (2.29) we have introduced the even-odd-odd intersection numbers

$$
\mathcal{I}_{a \alpha \beta} \equiv \int_{X} \omega_{a} \wedge \chi_{\alpha} \wedge \chi_{\beta}
$$

and the locally defined potentials $\kappa_{a}(z, \bar{z} ; v)$ such that

$$
\omega_{a}=\mathrm{i} \partial \bar{\partial} \kappa_{a}
$$

These potentials also enter the definition of $h(v)$ :

$$
h_{a}(v) \equiv-\frac{1}{4 \pi \ell_{\mathrm{s}}^{4}} \int_{X}\left(2 \pi \kappa_{a}-\log \left|\zeta_{a}\right|^{2}\right)\left(\frac{\mathrm{i}}{2 \operatorname{Im} \tau} G_{3} \wedge \bar{G}_{3}-Q_{6}^{\mathrm{nd}}\right)
$$

where $\zeta_{a}$ is the holomorphic section of the line bundle $\mathcal{O}\left(D_{a}\right)$ such that $D_{a}=\left\{\zeta_{a}=0\right\}$. By using the formula $\delta^{2}\left(D_{a}\right)=\frac{\mathrm{i}}{2 \pi} \partial \bar{\partial} \log \left|\zeta_{a}\right|^{2}$, this holomorphic section can also be used to write the last integral in (2.29) as an integral over the entire internal space $X$ :

$$
\int_{D_{a}}(\ldots)=\int_{X}(\ldots) \wedge \delta^{2}\left(D_{a}\right)=\frac{\mathrm{i}}{2 \pi} \int_{X}(\ldots) \wedge \partial \bar{\partial} \log \left|\zeta_{a}\right|^{2} .
$$

The last line in (2.29), which does not appear in the corresponding formula of [1], is the new contribution due to the hidden $G_{3}$ dependence on the Kähler moduli. As a non-trivial consistency condition, the definition (2.29) should not depend on the choice of the divisor $D_{a}$ within its equivalence class, up to possible $(\mathrm{hol}+\overline{\mathrm{hol}})$ contributions. This can be indeed verified by using (2.33) and, remarkably, the last line of (2.29) is actually crucial to prove this property once the $G_{3}$ dependence on the Kähler moduli is taken into account.

In the following we will need the derivatives of $\operatorname{Re} \rho_{a}$ with respect to the non-universal Kähler moduli $v^{a}$. These can be computed by adapting the corresponding calculation in [1]. In particular, a key formula proved in appendix A.1 of [1] is the following: under a generic variation $\delta v^{a}$ of the non-universal Kähler moduli, we have that

$$
\delta \kappa_{a}(y ; v)=2 \delta v^{b} \int_{X, y^{\prime}} G_{X}\left(y, y^{\prime}\right)\left(J \wedge \omega_{a} \wedge \omega_{b}\right)(y)
$$

where $G_{X}\left(y, y^{\prime}\right)=G_{X}\left(y^{\prime}, y\right)$ is the Green's function associated with the internal metric $\mathrm{d} s_{X}^{6}$. This Green's function can also be used to express $e^{-4 A_{0}}$ appearing in (2.5) as follows:

$$
e^{-4 A_{0}(y)}=\frac{1}{\ell_{\mathrm{s}}^{4}} \int_{X, y^{\prime}} G_{X}\left(y, y^{\prime}\right) Q_{6}\left(y^{\prime}\right) .
$$

By taking (2.33), (2.19) and (2.24) into account, together with (2.34) and (2.35), one can verify that the hidden dependence on the $v^{a}$ 's carried by the $G_{3}$ flux appearing in (2.32) and by the terms in the second line of (2.29) nicely combine, so that we obtain

$$
\delta \operatorname{Re} \rho_{a}=\mathcal{M}_{a b} \delta v^{b}
$$

with

$$
\mathcal{M}_{a b} \equiv \int_{X} e^{-4 A} J \wedge \omega_{a} \wedge \omega_{b}-\frac{1}{2 \operatorname{Im} \tau \ell_{\mathrm{s}}^{4}} \int_{X} \omega_{a} \wedge \operatorname{Re}\left(\Lambda_{b}^{1,0} \wedge \bar{G}_{3}\right)
$$


Notice that the matrix $\mathcal{M}_{a b}$ does not depend on the arbitrarily chosen divisors $D_{a}$ and is symmetric. To make the latter property manifest, one may use the fact that it does not depend on the possible $\partial$-exact contribution to $\Lambda_{b}^{1,0}$ either. One can then fix the gauge $\partial^{\dagger} \Lambda_{b}^{1,0}=0$ and use (2.21) to write the second integral on the right-hand side of (2.37) as

$$
\int_{X} \omega_{a} \wedge \operatorname{Re}\left(\Lambda_{b}^{1,0} \wedge \bar{G}_{3}\right)=\operatorname{Re} \int_{X} \bar{\partial} \Lambda_{a}^{1,0} \wedge *_{X} \partial \bar{\Lambda}_{b}^{0,1}
$$

Another useful property of $\mathcal{M}_{a b}$, which can be derived from (2.18) and (2.6), is the following:

$$
\left.\mathcal{M}_{a b} v^{b}=a \mathcal{I}_{a b c} v^{b} v^{c}=2 \mathrm{v}_{0} a(J\lrcorner \omega_{a}\right) .
$$

\subsection{Effective Lagrangian}

Having identified the real part of the chiral fields $\rho_{a}$, one can proceed with the calculation of the effective Lagrangian following from the Kähler potential (2.13). In particular, the bosonic effective Lagrangian for gravity and the moduli sector is given by

$$
\mathcal{L}_{\text {bos }}=\frac{1}{2} M_{\mathrm{P}}^{2} R_{4} * 1-M_{\mathrm{P}}^{2} K_{\mathcal{I} \overline{\mathcal{J}}} \mathrm{d} \varphi^{\mathcal{I}} \wedge * \mathrm{~d} \bar{\varphi}^{\overline{\mathcal{J}}}
$$

with $K_{\mathcal{I} \overline{\mathcal{J}}} \equiv \partial_{\mathcal{I}} \partial_{\overline{\mathcal{J}}} K \equiv \frac{\partial^{2} K}{\partial \varphi^{\mathcal{I}} \partial \bar{\varphi}^{\mathcal{J}}}$, where $\varphi^{\mathcal{I}}$ collectively denote the chiral fields $\left(\rho_{a}, \beta^{\alpha}, Z_{I}^{i}\right)$.

In principle, one may invert (2.29) and write the universal modulus $a$, and then the Kähler potential, as a function of $\left(\operatorname{Re} \rho_{a}, \operatorname{Im} \beta^{\alpha}, Z_{I}^{i}, \bar{Z}_{I}^{i}\right)$. However in general, unfortunately, it is not possible to find this function and so the general explicit form of the Kähler potential is not known, as it is also the case in the constant warping approximation [33]. Nevertheless, as discussed in appendix A.2, one can still compute the second derivatives of $K$ appearing in (2.40), along the lines of $[1,33]$. The resulting effective bosonic Lagrangian (2.40) takes the form

$$
\begin{aligned}
M_{\mathrm{P}}^{-2} \mathcal{L}_{\text {bos }}= & \frac{1}{2} R_{4} * 1-\mathcal{G}^{a b} \nabla \rho_{a} \wedge * \nabla \bar{\rho}_{b}+\frac{1}{4 \mathrm{v}_{0} a \operatorname{Im} \tau} v^{a} \mathcal{I}_{a \alpha \beta} \mathrm{d} \beta^{\alpha} \wedge * \mathrm{~d} \bar{\beta}^{\beta} \\
& -\frac{1}{2 \mathrm{v}_{0} a} \sum_{I} g_{i j}\left(Z_{I}, \bar{Z}_{I}\right) \mathrm{d} Z_{I}^{i} \wedge * \mathrm{~d} \bar{Z}_{I}^{\bar{\jmath}} .
\end{aligned}
$$

Here the kinetic metric $\mathcal{G}^{a b}$ is defined as follows

$$
\mathcal{G}^{a b} \equiv-\frac{1}{4 \mathrm{v}_{0} a}\left(\mathcal{M}^{a b}-\frac{1}{2 \mathrm{v}_{0} a} v^{a} v^{b}\right)
$$

where $\mathcal{M}^{a b}$ is the inverse of the matrix $\mathcal{M}_{a b}$ defined in (2.37), and we have introduced the covariant exterior derivative

$$
\nabla \rho_{a} \equiv \mathrm{d} \rho_{a}-\mathcal{A}_{a i}^{I} \mathrm{~d} Z_{I}^{i}-\frac{\mathrm{i}}{\operatorname{Im} \tau} \mathcal{I}_{a \alpha \beta} \operatorname{Im} \beta^{\alpha} \mathrm{d} \beta^{\beta}
$$

with

$$
\mathcal{A}_{a i}^{I} \equiv \frac{\partial \kappa_{a}\left(Z_{I}, \bar{Z}_{I} ; v\right)}{\partial Z_{I}^{i}}
$$


denoting the connection along the moduli space of the $I$-th D3-brane. Furthermore $g_{i \bar{\jmath}}(z, \bar{z})$ is the metric on the internal space and indeed the last line in (2.41) matches the kinetic terms obtained by expanding the action of probe D3-branes.

Notice also that the inverse of $\mathcal{G}^{a b}$ is given by

$$
\begin{aligned}
\mathcal{G}_{a b} & =-4 \mathrm{v}_{0} a \mathcal{M}_{a b}+\mathcal{M}_{a c} \mathcal{M}_{b d} v^{c} v^{d} \\
& =4 \mathrm{v}_{0} a\left[\int_{X} e^{-4 A} \omega_{a} \wedge *_{X} \omega_{b}+\frac{1}{2 \operatorname{Im} \tau \ell_{\mathrm{s}}^{4}} \int_{X} \omega_{a} \wedge \operatorname{Re}\left(\Lambda_{b}^{1,0} \wedge \bar{G}_{3}\right)\right] .
\end{aligned}
$$

Equation (2.38) shows that the second, manifestly $G_{3}$ dependent, term in the last line of $(2.45)$ is positive definite. Hence, such contribution tends to increase $\mathcal{G}_{a b}$ or, in other words, to suppress the kinetic metric $\mathcal{G}^{a b}$.

The effective Lagrangian derived here is almost identical to the Lagrangian derived in [1]. The only difference is that the matrix $\mathcal{M}_{a b}$ (denoted by $M_{\mathrm{w}}^{a b}$ in [1]) now contains also the manifestly $G_{3}$ dependent term appearing in (2.37). Furthermore, by looking at the kinetic terms for $\operatorname{Im} \rho_{a}$ in (2.41), we recognise the terms obtained in [22, 25] by freezing the non-universal Kähler moduli and focusing on the $C_{4}$-moduli, including such explicitly $G_{3}$ dependent contribution. Hence our effective theory provides the manifestly supersymmetric completion of the results of $[22,25]$, consistently combining the different possible moduli of this class of flux compactifications. Furthermore, it matches other previous partial results present in the literature, see the discussion in [1] and references therein.

The manifestly supersymmetric structure of our theory allows us to check that it satisfies the no-scale condition $[26,27]$

$$
K^{\mathcal{I} \overline{\mathcal{J}}} K_{\mathcal{I}} K_{\overline{\mathcal{J}}}=3
$$

where $K_{\mathcal{I}} \equiv \partial_{\mathcal{I}} K$ and $K^{\mathcal{I} \overline{\mathcal{J}}}$ is the inverse of $K_{\overline{\mathcal{I}} \mathcal{J}}$. As in [1], this provides a non-trivial check of the consistency of our theory, since the underlying ten-dimensional vacua are known to allow for a no-scale-like supersymmetry-breaking flux $G^{0,3} \neq 0$.

The no-scale condition (2.46) can be more easily verified by rewriting it as $\operatorname{det} A_{\mathcal{I} \overline{\mathcal{J}}}=0$, where $A_{\mathcal{I} \overline{\mathcal{J}}} \equiv \partial_{\mathcal{I}} \partial_{\overline{\mathcal{J}}} e^{-\frac{1}{3} K}=\partial_{\mathcal{I}} \partial_{\overline{\mathcal{J}}} a$. Indeed, by using (A.10) and (A.11) it is not difficult to see that, in our case, $\operatorname{det} A_{\mathcal{I} \overline{\mathcal{J}}} \propto \operatorname{det}\left(\frac{\partial v^{a}}{\partial \operatorname{Re} \rho_{b}}\right)=0$.

\subsection{Formulation with linear multiplets}

Our effective theory appears somewhat implicit, in the sense that different quantities are only implicitly defined in terms of the chiral fields $\rho_{a}, \beta^{\alpha}, Z_{I}^{i}$. However, its structure becomes more transparent if instead of the chiral multiplets $\rho_{a}$ we use as elementary fields their dual linear multiplets $l^{a}$ - see for instance $[34,35]$ for reviews on the chiral/linear duality.

Each linear multiplet $l^{a}$ contains a real scalar $l^{a}$ (denoted by same symbol) and a three-form field-strength $\mathcal{H}^{a}$ as bosonic components. The dualisation to linear multiplets is possible since the Kähler potential (2.13) does not depend on the axionic fields $\operatorname{Im} \rho_{a}$, which can then be dualised to the two-form potentials associated with the field-strengths 
$\mathcal{H}^{a}$. On the other hands the real scalars $l^{a}$ are given by

$$
l^{a}=-\frac{1}{2} \frac{\partial K}{\partial \operatorname{Re} \rho_{a}}=\frac{v^{a}}{2 \mathrm{v}_{0} a} .
$$

Hence, in the dual picture the universal modulus $a$ and the (constrained) non-universal Kähler moduli $v^{a}$ reorganise themselves into the $h_{+}^{1,1}$ linear multiplets $l^{a}$. Indeed (2.47) can be easily inverted into

$$
a=\frac{1}{2 \mathrm{v}_{0}}\left(\frac{6 \mathrm{v}_{0}}{\mathcal{I}_{a b c} l^{a} l^{b} l^{c}}\right)^{\frac{1}{3}}, \quad v^{a}=l^{a}\left(\frac{6 \mathrm{v}_{0}}{\mathcal{I}_{b c d} l^{b} l^{c} l^{d}}\right)^{\frac{1}{3}} .
$$

By using (2.48) one can obtain, through a Legendre transform, the kinetic potential

$$
\tilde{K} \equiv K+2 l^{a} \operatorname{Re} \rho_{a}
$$

which specifies the dual theory. Up to an irrelevant additional constant, this has the following explicit form

$$
\begin{aligned}
\tilde{K}= & \log \left(\mathcal{I}_{a b c} l^{a} l^{b} l^{c}\right)+\sum_{I} \hat{k}\left(Z_{I}, \bar{Z}_{I} ; l\right)-\frac{1}{\operatorname{Im} \tau} l^{a} \mathcal{I}_{a \alpha \beta} \operatorname{Im} \beta^{\alpha} \operatorname{Im} \beta^{\beta} \\
& -\frac{1}{2 \pi \ell_{\mathrm{s}}^{4}} \int_{X}\left[2 \pi \hat{k}-l^{a} \log \left|\zeta_{a}\right|^{2}\right]\left(\frac{\mathrm{i}}{2 \operatorname{Im} \tau} G_{3} \wedge \bar{G}_{3}-Q_{6}^{\mathrm{nd}}\right) \\
& -\frac{1}{\operatorname{Im} \tau \ell_{\mathrm{s}}^{4}} l^{a} \int_{D_{a}}\left[\operatorname{Re}\left(b^{1,0} \wedge \bar{G}_{3}\right)-\frac{1}{2} \bar{\partial} b^{1,0} \wedge \partial \bar{b}^{0,1}\right]
\end{aligned}
$$

where

$$
\hat{k}(z, \bar{z} ; l) \equiv l^{a} \kappa_{a}(z, \bar{z} ; l)
$$

can be considered as the Kähler potential associated with the rescaled Kähler form

$$
\hat{J} \equiv \mathrm{i} \partial \bar{\partial} \hat{k} \equiv \frac{1}{2 \mathrm{v}_{0} a} J=l^{a} \omega_{a}
$$

whose (unconstrained) Kähler moduli are given by the bosonic components of the linear multiplets.

The effective field theory can be derived from the kinetic potential (2.50), which must be considered as a function of $\left(l^{a}, \beta^{\alpha}, \bar{\beta}^{\bar{\alpha}}, Z_{I}^{i}, \bar{Z}_{I}^{\bar{i}}\right)$. In particular, the bosonic action is given by [35]

$$
\begin{aligned}
M_{\mathrm{P}}^{-2} \mathcal{L}_{\text {linear }}= & \frac{1}{2} R_{4} * 1+\frac{1}{4} \tilde{K}_{a b}\left(\mathrm{~d} l^{a} \wedge * \mathrm{~d} l^{b}+\mathcal{H}^{a} \wedge * \mathcal{H}^{b}\right)-\tilde{K}_{\mathcal{I} \overline{\mathcal{J}}} \mathrm{d} \varphi^{\mathcal{I}} \wedge * \mathrm{~d} \bar{\varphi}^{\overline{\mathcal{J}}} \\
& +\frac{\mathrm{i}}{2}\left(\tilde{K}_{a \mathcal{I}} \mathrm{d} \varphi^{\mathcal{I}}-\tilde{K}_{a \overline{\mathcal{I}}} \mathrm{d} \overline{\overline{\mathcal{I}}}\right) \wedge \mathcal{H}^{a}
\end{aligned}
$$

where now $\varphi^{\mathcal{I}}$ collectively denote only the chiral fields $\left(\beta^{\alpha}, Z_{I}^{i}\right)$ and we have used the notation for derivatives introduced in (2.46), e.g. $\tilde{K}_{a \mathcal{I}} \equiv \frac{\partial^{a} \tilde{K}}{\partial l^{a} \partial \varphi^{\mathcal{I}}}$, etc. Notice that while computing the derivatives of $\tilde{K}$ one should again take into account the hidden dependence of $G_{3}$ on the Kähler moduli and hence on the scalars $l^{a}$. Since the primitivity condition (2.12) 
is unaffected by a possible rescaling of the Kähler form, the discussion of section 2.1 can be easily rephrased by replacing $J \rightarrow \hat{J}$ and the formulas (2.19) and (2.24) can be rewritten as

$$
\frac{\partial G_{3}}{\partial l^{a}}=\partial \bar{\partial} \hat{\Lambda}_{a}^{1,0}, \quad \frac{\partial b^{1,0}}{\partial l^{a}}=\hat{\Lambda}_{a}^{1,0}
$$

respectively, with $\hat{\Lambda}_{a}^{1,0} \equiv 2 \mathrm{v}_{0} a \Lambda_{a}^{1,0}$ such that

$$
\hat{\Delta}_{X} \hat{\Lambda}_{a}^{1,0}=-2 \hat{*}_{X}\left(\omega_{a} \wedge G_{3}\right) .
$$

Here and in the following all 'hatted' operators are defined in terms of the rescaled Kähler metric

$$
\mathrm{d} \hat{s}_{X}^{2} \equiv \frac{1}{2 \mathrm{v}_{0} a} \mathrm{~d} s_{X}^{2}
$$

associated with the rescaled Kähler potential (2.52).

The derivatives appearing in $(2.53)$ can be explicitly computed and $\mathcal{L}_{\text {linear }}$ assumes the following form

$$
\begin{aligned}
M_{\mathrm{P}}^{-2} \mathcal{L}_{\text {linear }}= & \frac{1}{2} R_{4} * 1-\frac{1}{4} \mathcal{G}_{a b}\left(\mathrm{~d} l^{a} \wedge * \mathrm{~d} l^{b}+\mathcal{H}^{a} \wedge * \mathcal{H}^{b}\right) \\
& -\sum_{I} \hat{g}_{i \bar{\jmath}}\left(Z_{I}, \bar{Z}_{I}\right) \mathrm{d} Z_{I}^{i} \wedge * \mathrm{~d} \bar{Z}_{I}^{\bar{\jmath}}+\frac{1}{2 \operatorname{Im} \tau} l^{a} \mathcal{I}_{a \alpha \beta} \mathrm{d} \beta^{\alpha} \wedge * \mathrm{~d} \bar{\beta}^{\beta} \\
& -\operatorname{Im}\left(\mathcal{A}_{a i}^{I} \mathrm{~d} Z_{I}^{i}+\frac{\mathrm{i}}{\operatorname{Im} \tau} \mathcal{I}_{a \alpha \beta} \operatorname{Im} \beta^{\alpha} \mathrm{d} \beta^{\beta}\right) \wedge \mathcal{H}^{a}
\end{aligned}
$$

In this formula, $\mathcal{G}_{a b}$ is the same matrix introduced in (2.45), which can be rewritten as follows

$$
\mathcal{G}_{a b}=2 \int_{X} e^{-4 \hat{A}} \omega_{b} \wedge \hat{*}_{X} \omega_{b}+\frac{1}{\operatorname{Im} \tau \ell_{\mathrm{s}}^{4}} \int_{X} \omega_{a} \wedge \operatorname{Re}\left(\hat{\Lambda}_{b}^{1,0} \wedge \bar{G}_{3}\right)
$$

where the rescaled warp factor

$$
e^{-4 \hat{A}} \equiv\left(2 v_{0} a\right)^{2} e^{-4 A}=\frac{3}{\mathcal{I}_{a b c} l^{a} l^{b} l^{c}}+e^{-4 \hat{A}_{0}}
$$

solves the modified equation obtained by substituting the metric (2.56) in (2.2), while $e^{-4 \hat{A}_{0}}$ is the particular solution with vanishing total integral.

Clearly the use of linear multiplets allows for a more explicitly formulation of the effective theory in terms of the elementary fields, which furthermore have a more direct connection with the geometrical structure of the underlying ten-dimensional vacua. In particular, the rescaled Kähler metric (2.56) can be interpreted precisely as the metric 'seen' by the mobile D3-branes and naturally enters the other quantities describing the effective theory. In this sense, at least at the perturbative level, the linear multiplet formulation appears more natural than the corresponding formulation in terms of chiral multiplets.

\section{Effective theory of warped M-theory models}

So far we have assumed the axion-dilaton $\tau$ to be constant, but the results should clearly extend to F-theory models, i.e. with non-constant holomorphic $\tau$ and bulk seven-branes. A 
purely IIB description of such generalisation is complicated by the fact that $G_{3}$ transforms non-trivially under $\mathrm{SL}(2 ; \mathbb{Z})$ duality transformations. Furthermore, once seven-branes are introduced, the associated world-volume fluxes are naturally entangled with the $G_{3}$ fluxes through the Bianchi identities and must themselves satisfy a primitivity condition. The cohomological structures describing these effects must be appropriately 'twisted' in order to take in account the non-trivial $\mathrm{SL}(2 ; \mathbb{Z})$ transformations and are then more complicated with respect to the constant $\tau$ case considered in the previous section.

On the other hand, these technical difficulties appear more treatable if addressed from the dual perspective of M-theory flux compactifications to three dimensions [29, 30] on elliptically fibered Calabi-Yau four-folds. In such a dual description, the holomorphic axion-dilaton and the seven-branes are geometrised into the non-triviality of the elliptic fibration [28] and the IIB three-form and seven-brane fluxes are both represented by the M-theory four-form flux.

In the following we will show how the same logic followed for type IIB in the section 2 and in [1] can be easily adapted to the M-theory vacua described in [29, 30], by considering flux compactifications on generic, non-necessarily elliptically fibered, Calabi-Yau four-folds. The effective theory for these kinds of compactifications was first obtained in the constant warping approximation in $[36,37],{ }^{5}$ while the effect of a weak warping and of higher derivative corrections has been more recently studied in [23, 24]. As we will see, our approach allows us to incorporate the effect of the four-form flux, mobile M2-branes and of a possibly strong warping in a consistent way. We will discuss a simple duality check of consistency between the M-theory and IIB results, leaving a detailed study of the implications of our results for F-theory models to the future.

\subsection{Eleven-dimensional structure}

In the M-theory flux vacua of $[29,30]$ the metric takes the form

$$
\mathrm{d} s_{11}^{2}=\ell_{\mathrm{M}}^{2}|\Phi|^{4} e^{4 D} \mathrm{~d} s_{3}^{2}+\ell_{\mathrm{M}}^{2} e^{-2 D} \mathrm{~d} s_{Y}^{2}
$$

where $\ell_{\mathrm{M}}$ is the M-theory Planck length, $\mathrm{d} s_{3}^{2}$ is the flat three-dimensional metric, $\mathrm{d} s_{Y}^{2}$ is the Ricci-flat metric of a Calabi-Yau four-fold $Y$ and the warp factor $e^{D}$ varies along $Y$. Furthermore $\Phi$ (which has dimension [mass $]^{\frac{1}{2}}$ ) is a constant playing the role of conformal compensator and is fixed by the three-dimensional Einstein frame condition.

The M-theory field-strength $F_{4}$ has the form

$$
F_{4}=\ell_{\mathrm{M}}^{3}|\Phi|^{6} \mathrm{dvol}_{3} \wedge \mathrm{d} e^{6 D}+G_{4}
$$

where $\mathrm{dvol}_{3}$ is the volume form associated with $\mathrm{d} s_{3}^{2}$. The internal $G_{4}$ flux must be self-dual, $*_{Y} G_{4}=G_{4}$. More precisely, three-dimensional $\mathcal{N}=2$ supersymmetry requires the internal $G_{4}$ flux to be purely $(2,2)$ and primitive,

$$
J \wedge G_{4}=0
$$

\footnotetext{
${ }^{5}$ See also [38] for a discussion relevant for applications to F-theory models.
} 
while the possible $(4,0)$ and $(0,4)$ components of $G_{4}$, also allowed by the self-duality condition, break supersymmetry in a no-scale way. In addition, these vacua can host mobile M2-branes.

Under the above conditions, the equation of motion of $G_{4}$ implies that the warp factor is determined by the Poisson equation

$$
\Delta_{Y} e^{-6 D}=\frac{1}{\ell_{\mathrm{M}}^{6}} *_{Y} Q_{8}
$$

where $Q_{8}$ is the M2-charge density

$$
Q_{8}=\frac{1}{2} G_{4} \wedge G_{4}+\ell_{\mathrm{M}}^{6} \sum_{I \in \mathrm{M} 2} \delta_{I}^{8}-\ell_{\mathrm{M}}^{6} I_{8} .
$$

Here $I_{8}$ is a closed 8-form that is quartic in the curvature and integrates to $\frac{1}{24}$ the Euler characteristic of $Y: \int_{Y} I_{8}=\frac{1}{24} \chi(Y)$ [39]. As in the IIB case, once the tadpole condition $\int_{X} Q_{8}=0$ is satisfied, the general solution of (3.4) can be written as

$$
e^{-6 D}=c+e^{-6 D_{0}}
$$

where $c$ is the universal modulus of these M-theory compactifications - the counterpart of $a$ in type IIB - and $e^{-6 D_{0}}$ is the particular solution of (3.4) that satisfies the condition

$$
\int_{Y} e^{-6 D_{0}} \mathrm{dvol}_{Y}=0
$$

The universal modulus is the breathing mode of the internal space, while the volume of the internal space is not physical and can be fixed to the any given (dimensionless) value

$$
\int_{Y} \mathrm{dvol}_{Y}=\frac{1}{4 !} \int_{Y} J \wedge J \wedge J \wedge J \equiv \mathrm{w}_{0} .
$$

\subsection{Kähler potential}

The arguments followed in [1] and reviewed in section 2 can be naturally adapted to the warped M-theory compactifications and lead to similar results. So, we will be sketchy. First, we assume that the $G_{4}$-flux stabilises completely the complex structure moduli of $Y$ (i.e. the IIB axion-dilaton, complex structure and seven brane moduli in an F-theory context). One can then invoke the same arguments based on superconformal invariance of $[1,5,6]$, for instance by using the super-Weyl invariant three-dimensional supergravity described in detail in [40]. In particular, the three-dimensional Einstein term takes the form

$$
\frac{1}{2} C \int \sqrt{-g_{3}}|\Phi|^{2} e^{-\frac{1}{4} K} R_{3}
$$

where $K$ denotes the three-dimensional Kähler potential and $C$ is an arbitrary positive constant which may be reabsorbed into a rescaling of $\Phi$. By matching (3.9) with the expression obtained by dimensionally reducing the M-theory action leads to the identification

$$
C e^{-\frac{1}{4} K}=4 \pi \int_{Y} e^{-6 D} \mathrm{dvol}_{Y}=4 \pi \mathrm{w}_{0} c
$$


where, in the second step, we have used the splitting (3.6) and the normalisation condition (3.7). Hence, by choosing $C=4 \pi \mathrm{w}_{0}$, we conclude that the three-dimensional Kähler potential takes the following simple form

$$
K=-4 \log c
$$

which is completely analogous to (2.13). Fixing the super-Weyl symmetry and imposing a canonical Einstein-Hilbert term $\frac{1}{2} M_{\mathrm{P}} \int \sqrt{-g_{3}} R_{3}$ require that

$$
\Phi=\left(\frac{M_{\mathrm{P}}}{4 \pi \mathrm{w}_{0}}\right)^{\frac{1}{2}} e^{\frac{1}{8} K}=\left(\frac{M_{\mathrm{P}}}{4 \pi \mathrm{w}_{0} c}\right)^{\frac{1}{2}} .
$$

\subsection{Kähler moduli and $G_{4}$ dependence}

The Calabi-Yau Kähler form $J$ can be expanded as follows

$$
J=u^{A} \omega_{A} .
$$

Here $\omega_{A}$ are harmonic $(1,1)$-forms defining a basis of $H^{2}(Y ; \mathbb{Z})$ and $u^{A}$ are the non-universal Kähler parameters which, because of (3.8), must satisfy the constraint

$$
\frac{1}{4 !} \mathcal{I}_{A B C D} u^{A} u^{B} u^{C} u^{D}=\mathrm{w}_{0}
$$

where we have introduced the intersection numbers

$$
\mathcal{I}_{A B C D} \equiv \int_{Y} \omega_{A} \wedge \omega_{B} \wedge \omega_{C} \wedge \omega_{D}
$$

In the following, we will also use local potentials $\kappa_{A}$ such that

$$
\omega_{A}=\mathrm{i} \partial \bar{\partial} \kappa_{A} .
$$

Geometrically, $e^{-2 \pi \kappa_{A}}$ defines a metric for the line bundle $\mathcal{O}\left(S_{A}\right)$.

The actual (non-massive) Kähler moduli must respect (3.3). This condition can be imposed at a cohomological level by requiring that

$$
u^{A}\left[\omega_{A} \wedge G_{4}\right]=0 \quad \text { in } H^{6}(Y) .
$$

The $u^{A}$ 's preserving (3.17) can be then parametrised in terms of the actual Kähler moduli $v^{a}, a=1, \ldots, N_{K}$, as follows

$$
u^{A}=m_{a}^{A} v^{a}
$$

where $\vec{m}_{a}=\left(m_{a}^{1}, \ldots, m_{a}^{b_{2}(Y)}\right)$ provide a set of $N_{K}$ independent vectors such that

$$
m_{a}^{A}\left[\omega_{A} \wedge G_{4}\right]=0
$$

Since $G_{4}+\frac{1}{2} c_{2}(Y)$, where $c_{2}(Y)$ is the second Chern class of $Y$, defines an element of $H^{4}(Y ; \mathbb{Z})[41]$, the numbers $m_{a}^{A}$ can be chosen to be integral: $m_{a}^{A} \in \mathbb{Z}$. 
One can now repeat the arguments of section (2.1) and deduce that a deformation $\delta v^{a}$ must be accompanied by a deformation of the $G_{4}$ flux. The corresponding variation $\delta G_{4}$ can be written as

$$
\delta G_{4}=\mathrm{i} \delta v^{a} \partial \bar{\partial} \Lambda_{a}^{1,1}
$$

where $\Lambda_{a}^{1,1}$ are real $(1,1)$-forms, defined up to harmonic and $\partial \bar{\partial}$-exact pieces. Each $\Lambda_{a}^{1,1}$ must satisfy

$$
v^{a} \Lambda_{a}^{1,1}=0
$$

up to harmonic and $\partial \bar{\partial}$-exact pieces, and

$$
\mathrm{i} \partial \bar{\partial} \Lambda_{a}^{1,1} \wedge J=-\omega_{a} \wedge G_{4}
$$

where

$$
\omega_{a} \equiv m_{a}^{A} \omega_{A} .
$$

We can impose that the harmonic component of $\Lambda_{a}^{1,1}$ vanishes:

$$
\left(\Lambda_{a}^{1,1}\right)_{\text {harm }}=0
$$

and fix the residual degeneracy by requiring that $\mathrm{d}^{\dagger} \Lambda_{a}^{1,1}=\partial^{\dagger} \Lambda_{a}^{1,1}=\bar{\partial}^{\dagger} \Lambda_{a}^{1,1}=0$. Under these conditions, the primitivity of $G_{4}$ implies that $\left.\Delta_{Y}(J\lrcorner \Lambda_{a}^{1,1}\right)=0$ and then $\Lambda_{a}^{1,1}$ is primitive itself: ${ }^{6}$

$$
J\lrcorner \Lambda_{a}^{1,1}=0 .
$$

In turn this property can be used to rewrite (3.22) in the form

$$
\Delta_{Y} \Lambda_{a}^{1,1}=-2 *_{Y}\left(\omega_{a} \wedge G_{4}\right) .
$$

Note that (3.21) is not an independent condition but, because of the primitivity of $G_{4}$, it is actually required by $(3.26)$.

Along the same lines, we can split

$$
G_{4}=G_{4}^{(0)}+\mathrm{i} \partial \bar{\partial} \mathcal{C}^{1,1}
$$

where $G_{4}^{(0)}$ is any fixed element of $H^{4,0}(Y) \oplus H^{2,2}(Y) \oplus H^{0,4}(Y)$ in the same class of $G_{4}$ while $\mathcal{C}^{1,1}$ is a globally defined real $(1,1)$-form encoding the Kähler moduli dependence:

$$
\delta \mathcal{C}^{1,1}=\delta v^{a} \Lambda_{a}^{1,1}
$$

We can then locally write $G_{4}^{(0)}=\mathrm{d} C_{3}^{(0)}$ and $G_{4}=\mathrm{d} C_{3}$ with

$$
C_{3}=C_{3}^{(0)}-\frac{\mathrm{i}}{2} \partial \mathcal{C}^{1,1}+\frac{\mathrm{i}}{2} \bar{\partial} \mathcal{C}^{1,1}+\ell_{\mathrm{M}}^{3}\left(\beta^{\alpha} \lambda_{\alpha}+\bar{\beta}^{\bar{\alpha}} \bar{\lambda}_{\bar{\alpha}}\right)
$$

where the $\lambda_{\alpha}, \alpha=1, \ldots, b_{3}(Y)$, define a harmonic basis of $H^{1,2}(Y)$.

\footnotetext{
${ }^{6}$ Indeed $\left.\Delta_{Y}(J\lrcorner \Lambda_{a}^{1,1}\right)=0$ implies that $\left.J\right\lrcorner \Lambda_{a}^{1,1}$ is a constant. On the other hand, by our gauge-fixing conditions, $\Lambda_{a}^{1,1}$ is a $\mathrm{d}^{\dagger}$-exact 2 -form. Since $\left.[J\lrcorner, \mathrm{d}^{\dagger}\right]=0$, also $\left.J\right\lrcorner \Lambda_{a}^{1,1}$ is $\mathrm{d}^{\dagger}$-exact and then it cannot be a non-vanishing constant.
} 


\subsection{Chiral coordinates}

We now need to describe the moduli space in chiral coordinates. The M2-brane moduli can be parametrised by their positions $Z_{I}^{i}$ in some local complex coordinates $z^{i}$ along $Y$. The moduli of the $C_{3}$ gauge potential can be identified with the $\beta^{\alpha}$ appearing in (3.29). On the other hand, the precise form of the chiral coordinates $\rho_{a}$ of the Kähler and $C_{6}$ moduli (where $C_{6}$ is the potential of $F_{7}=*_{11} F_{4}$ ) is less obvious, as for the Kähler $/ C_{4}$ moduli in type IIB.

One can proceed as in [1] and in section 2.2. The relevant instantons are given by Euclidean M5-branes. As in type IIB, the perturbative effective Lagrangian does not depend on the $C_{6}$ axions, and hence on $\operatorname{Im} \rho_{a}$. We can then focus on $\operatorname{Re} \rho_{a}$, which must be detected by the real part of the action of a probe supersymmetric M5-brane wrapping an effective divisor $S \subset Y$. This contains a warped volume contribution proportional to

$$
\frac{1}{3 !} \int_{S} e^{-6 D} J \wedge J \wedge J
$$

analogous to the first term on the right-hand side of (2.25). Hence, $\operatorname{Re} \rho_{a}$ must contain a contribution similar to (3.30) for an appropriately chosen divisor $S$. Indeed, this parametrisation has been used in [24] and shown to be consistent with a direct dimensional reduction.

More precisely, the contribution of (3.30) to $\operatorname{Re} \rho_{a}$ can be obtained by choosing the integration divisor

$$
S_{a}=m_{a}^{A} S_{A}
$$

where $S_{A}, A=1, \ldots, b_{2}(Y)$, are a set of divisors which are Poincaré dual to the integral harmonic forms $\omega_{A}$. As shown in appendix B.1, by discarding some $(\mathrm{hol}+\overline{\mathrm{hol}})$ contribution one is then led to identify part of $\operatorname{Re} \rho_{a}$ with

$$
\frac{1}{3 !} c \mathcal{I}_{a A B C} u^{A} u^{B} u^{C}+\frac{1}{2} \sum_{I} \kappa_{a}\left(Z_{I}, \bar{Z}_{I} ; v\right)+h_{a}(v)
$$

where $\mathcal{I}_{a A B C} \equiv m_{a}^{D} \mathcal{I}_{A B C D}, \kappa_{a}(z, \bar{z} ; v) \equiv m_{a}^{A} \kappa_{A}(z, \bar{z} ; v)$ [see equation (3.16)] and $h_{a}(v)$ is defined as follows

$$
h_{a}(v) \equiv \frac{1}{4 \pi \ell_{\mathrm{M}}^{6}} \int_{Y}\left(2 \pi \kappa_{a}-\log \left|\zeta_{a}\right|^{2}\right)\left[\frac{1}{2} G_{4} \wedge G_{4}-\ell_{\mathrm{M}}^{6} I_{8}\right]
$$

where $\zeta_{a}(z)$ is a holomorphic section of $\mathcal{O}\left(S_{a}\right)$ which vanishes on $S_{a}$.

The M5-brane action contains other terms in addition to (3.30). First, there may be higher order derivative corrections, similar to the $I_{8}$ appearing in (3.33), which may carry a dependence on the moduli, in particular on the Kähler ones. Such higher order corrections are not the main focus of the present paper and so we will neglect them, while considering $I_{8}$ as a non-dynamical contribution to the M2-charge density. One may incorporate their effect along the lines of $[23,24]$ which showed how corrections of this kind are in fact necessary in order to accommodate M-theory higher derivative contributions to the effective theory.

Rather, we concentrate on the $G_{4}$ dependent contribution to the M5-brane action, which is present already at the lowest derivative level. This term should arise from the contribution of the world-volume 3-form flux $T_{3}=\mathrm{d} A_{2}+C_{3}$ supported on the M5-brane which 
is, however, self-dual and then does not admit a simple Lagrangian description [42-44]. One can then follow a simpler strategy, which identifies such a term by requiring that, as in the type IIB case, the definition of $\operatorname{Re} \rho_{a}$ depends on the choice of the divisors $S_{A}$ (within their linear equivalence classes) at most by a $(\mathrm{hol}+\overline{\mathrm{hol}})$ term and matches the IIB result under duality. These conditions lead to the definition

$$
\begin{aligned}
\operatorname{Re} \rho_{a} \equiv & \frac{1}{3 !} c \mathcal{I}_{a A B C} u^{A} u^{B} u^{C}+\frac{1}{2} \sum_{I} \kappa_{a}\left(Z_{I}, \bar{Z}_{I} ; v\right)+h_{a}(v) \\
& +\frac{1}{2} \mathcal{T}_{a \alpha \bar{\beta}} \beta^{\alpha} \bar{\beta}^{\bar{\beta}}+\frac{1}{2 \ell_{\mathrm{M}}^{6}} \int_{S_{a}}\left(\mathcal{C}^{1,1} \wedge G_{4}+\frac{\mathrm{i}}{2} \partial \mathcal{C}^{1,1} \wedge \bar{\partial} \mathcal{C}^{1,1}\right)
\end{aligned}
$$

where

$$
\mathcal{T}_{a \alpha \bar{\beta}} \equiv \mathrm{i} \int_{Y} \omega_{a} \wedge \lambda_{\alpha} \wedge \bar{\lambda}_{\bar{\beta}}
$$

See subsection 3.6 below for a discussion on the matching with type IIB side.

Relegating the details of the calculation to appendix B.2, we obtain that, under a deformation of the non-universal Kähler moduli $\delta v^{a}$ [preserving (3.14) and (3.17)], $\operatorname{Re} \rho_{a}$ transforms as follows

$$
\delta \operatorname{Re} \rho_{a}=\mathcal{N}_{a b} \delta v^{b}
$$

where

$$
\mathcal{N}_{a b} \equiv \frac{1}{2} \int_{Y} e^{-6 D} J \wedge J \wedge \omega_{a} \wedge \omega_{b}+\frac{1}{2 \ell_{\mathrm{M}}^{6}} \int_{Y} \omega_{a} \wedge \Lambda_{b}^{1,1} \wedge G_{4}
$$

Note that, in $\delta \operatorname{Re} \rho_{a}$, the contributions due to the $G_{4}$ dependence on the Kähler moduli coming from $h_{a}(v)$ and from the second line of (3.34) nicely combine, so that the final result does not depend on the arbitrarily chosen divisors $S_{a}$.

We observe that equation (3.26) can be used to deduce that $\mathcal{N}_{a b}$ contains the manifestly symmetric and positive definite contribution

$$
-\frac{1}{2 \ell_{\mathrm{M}}^{6}} \int_{Y} \Lambda_{a}^{1,1} \wedge \omega_{b} \wedge G_{4}=\frac{1}{4 \ell_{\mathrm{M}}^{6}} \int_{Y} \mathrm{~d} \Lambda_{a}^{1,1} \wedge *_{Y} \mathrm{~d} \Lambda_{b}^{1,1}
$$

Note also that by contracting (3.37) with $v^{a}$ and using the primitivity of $G_{4}$ one gets the useful identity

$$
\left.\mathcal{N}_{a b} v^{b}=\frac{1}{2} c \mathcal{I}_{a A B C} u^{A} u^{B} u^{C}=3 \mathrm{w}_{0} c(J\lrcorner \omega_{a}\right) .
$$

\subsection{Effective Lagrangian}

By using the above results, one can compute the effective Lagrangian associated with the Kähler potential (3.11), as we did in section 2.3 for the type IIB case. The formula (2.40) is valid also for three-dimensional $\mathcal{N}=2$ theories, up to substituting $M_{\mathrm{P}}^{2}$ with $M_{\mathrm{P}}$, see for instance [40]. Hence, by using the results of appendix B.3, one obtains the following Lagrangian for the moduli sector of the effective theory:

$$
\begin{aligned}
M_{\mathrm{P}}^{-1} \mathcal{L}_{\mathrm{M}}= & \frac{1}{2} R_{4} * 1-\mathcal{G}^{a b} \nabla \rho_{a} \wedge * \nabla \bar{\rho}_{b}-\frac{1}{2 \mathrm{w}_{0} c} v^{a} \mathcal{T}_{a \alpha \bar{\beta}} \mathrm{d} \beta^{\alpha} \wedge * \mathrm{~d} \bar{\beta}^{\bar{\beta}} \\
& -\frac{1}{2 \mathrm{w}_{0} c} \sum_{I} g_{i \bar{\jmath}}\left(Z_{I}, \bar{Z}_{I}\right) \mathrm{d} Z_{I}^{i} \wedge * \mathrm{~d} \bar{Z}_{I}^{\bar{j}}
\end{aligned}
$$


We have introduced the covariant derivative

$$
\nabla \rho_{a} \equiv \mathrm{d} \rho_{a}-\mathcal{A}_{a i}^{I} \mathrm{~d} Z_{I}^{i}-\mathcal{T}_{a \alpha \bar{\beta}} \bar{\beta}^{\bar{\beta}} \mathrm{d} \beta^{\alpha}
$$

where

$$
\mathcal{A}_{a i}^{I} \equiv \frac{\partial \kappa_{a}\left(Z_{I}, \bar{Z}_{I} ; v\right)}{\partial Z_{I}^{i}}
$$

is the connection along the moduli space of the $I$-th M2-brane. The kinetic metric $\mathcal{G}^{a b}$ is defined as follows

$$
\mathcal{G}^{a b} \equiv-\frac{1}{4 \mathrm{w}_{0} c}\left(\mathcal{N}^{a b}-\frac{1}{3 \mathrm{w}_{0} c} v^{a} v^{b}\right)
$$

where $\mathcal{N}^{a b}$ is the inverse of the matrix $\mathcal{N}_{a b}$ defined in (3.37). Furthermore, note that the contribution appearing in the last line of (3.40) contains the Calabi-Yau metric $g_{i \bar{\jmath}}$ and perfectly matches the kinetic terms obtained by considering probe M2-branes on the M-theory vacua described in section (3.1).

The kinetic metric $\mathcal{G}^{a b}$ is the inverse of

$$
\begin{aligned}
\mathcal{G}_{a b} & =-4 \mathrm{w}_{0} c \mathcal{N}_{a b}+\frac{4}{9} \mathcal{N}_{a c} \mathcal{N}_{b d} v^{c} v^{d} \\
& =4 \mathrm{w}_{0} c\left(\int_{Y} e^{-6 D} \omega_{a} \wedge *_{Y} \omega_{b}-\frac{1}{2 \ell_{\mathrm{M}}^{6}} \int_{Y} \Lambda_{a}^{1,1} \wedge \omega_{b} \wedge G_{4}\right) .
\end{aligned}
$$

The first warped term in the second line was also obtained in $[23,24]$ by a direct dimensional reduction in a weak warping regime. However, as it happens in the IIB case, we see that it is valid also for a possible strong warping and that it must be completed by a $G_{4}$ dependent contribution. Note that such a contribution can be written in a manifestly symmetric and positive definite form by using (3.38). Hence, as in type IIB, this contribution of the flux tends to lower the value of the kinetic matrix $\mathcal{G}^{a b}$.

This supersymmetric theory satisfies the three-dimensional no-scale condition ${ }^{7}$

$$
K^{\mathcal{I} \overline{\mathcal{J}}} K_{\mathcal{I}} K_{\overline{\mathcal{J}}}=4
$$

where $K^{\mathcal{I} \overline{\mathcal{J}}}$ is the inverse of $K_{\overline{\mathcal{I}} \mathcal{J}}$ and we are adopting the usual notation: $K_{\mathcal{I}} \equiv \partial_{\mathcal{I}} K \equiv$ $\frac{\partial K}{\partial \varphi^{\mathcal{I}}}, K_{\mathcal{I} \overline{\mathcal{J}}} \equiv \partial_{\mathcal{I}} \partial_{\overline{\mathcal{J}}} K$ etc., with $\varphi^{\mathcal{I}}=\left(\rho_{a}, Z_{I}^{i}, \beta^{\alpha}\right)$. This is indeed consistent with the possibility of having supersymmetry breaking components $G^{4,0}+G^{0,4} \neq 0$, which would induce a non-vanishing contribution to the superpotential of the three-dimensional theory [45]. The condition (3.45) ensures the vanishing of the vacuum expectation value of the threedimensional potential - see for instance [40] for the component form of the action.

Finally, one may highlight the geometrical interpretation of the effective theory by dualising the chiral multiplets $\rho_{a}$ into vector multiplets. We do not present the details of such a dualisation, which would be the three-dimensional analog of what was presented in section 2.4 for type IIB compactifications to four dimensions. A more explicit discussion on this duality in our same context can be found in [24].

\footnotetext{
${ }^{7}$ The no-scale property can be more easily checked by verifying that the matrix $A_{\mathcal{I} \overline{\mathcal{J}}} \equiv \partial_{\mathcal{I}} \partial_{\overline{\mathcal{J}}} e^{-\frac{1}{4} K} \equiv$ $\partial_{\mathcal{I}} \partial_{\overline{\mathcal{J}}} c$ is degenerate.
} 


\subsection{Matching with type IIB}

By taking elliptically fibered Calabi-Yau fourfolds and $G_{4}$-fluxes satisfying appropriate transversality conditions, the M-theory flux compactifications discussed in the present section are dual to type IIB F-theory compactifications to four dimensions. Leaving to the future a detailed discussion on the implications of our results in the context of F-theory compactifications, we now give just a simple check of the compatibility between the Mtheory results of the present section and the IIB results of section 2 .

Indeed, one can locally assume an approximately factorised structure $Y \simeq X \times T_{\tau}^{2}$, where $T_{\tau}^{2}$ is a two-dimensional torus with approximately constant complex structure $\tau$. In such a case the duality relations between the M-theory and IIB (local) quantities are easily formulated, see for instance [2]. In particular, we are interested in the duality relation between the terms involving the $G_{3} / G_{4}$ fluxes in the definitions of $\rho_{a}$ in type IIB/M-theory respectively.

Writing the $T_{\tau}^{2}$ metric as

$$
\mathrm{d} s_{T_{\tau}^{2}}^{2}=\frac{L^{2}}{\operatorname{Im} \tau} \mathrm{d} z \mathrm{~d} \bar{z}
$$

where $z=x+\tau y$, with periodicities $x \simeq x+1$ and $y \simeq y+1$, the above factorised structure corresponds to taking $\mathrm{d} s_{Y}^{2} \simeq \mathrm{d} s_{X}^{2}+\mathrm{d} s_{T_{\tau}^{2}}^{2}$. $L^{2}$ represents the volume of $T_{\tau}^{2}$ which is eventually sent to zero in the F-theory limit. Then, as in [2], the M-theory/IIB $G_{4} / G_{3}$ fluxes are related as follows

$$
G_{4}=\frac{L}{\operatorname{Im} \tau} \operatorname{Im}\left(\mathrm{d} \bar{z} \wedge G_{3}\right) .
$$

By consistency, the relation between $b^{1,0}$ and $\mathcal{C}^{1,1}$ appearing in (2.22) and (3.29), respectively, is ${ }^{8}$

$$
\mathcal{C}^{1,1}=-\frac{L}{\operatorname{Im} \tau} \operatorname{Re}\left(\mathrm{d} \bar{z} \wedge b^{1,0}\right) .
$$

Let us now choose as M-theory divisors $S_{a}$ the vertical divisors in $Y$ obtained by 'attaching' the $T_{\tau}^{2}$ fibre over the divisors $D_{a}$ in the base $X$. Then, by plugging (3.47) and (3.48) into the last term appearing in the definition (3.34) of $\operatorname{Re} \rho_{a}$ and performing the integration over the $T_{\tau}^{2}$ fibre in $S_{a}$, it is easy to check that

$$
\frac{1}{2 \ell_{\mathrm{M}}^{6}} \int_{S_{a}}\left(\mathcal{C}^{1,1} \wedge G_{4}+\frac{\mathrm{i}}{2} \partial \mathcal{C}^{1,1} \wedge \bar{\partial} \mathcal{C}^{1,1}\right)=-\frac{L^{2}}{2 \ell_{\mathrm{M}}^{6} \operatorname{Im} \tau} \int_{D_{a}}\left[\operatorname{Re}\left(b^{1,0} \wedge \bar{G}_{3}\right)-\frac{1}{2} \bar{\partial} b^{1,0} \wedge \partial \bar{b}^{0,1}\right]
$$

Upon using the identification $\ell_{\mathrm{M}}^{3}=L \ell_{\mathrm{s}}^{2}$ [2], we see that (3.49) exactly reproduces the last term in the definition (2.29) of $\operatorname{Re} \rho_{a}$ in type IIB. This shows how such contributions, which are directly related to the manifestly flux-dependent contributions to the respective effective Lagrangians, are indeed perfectly compatible under duality. Similar consistency checks for the other terms appearing in (2.29) and (3.34) can also be performed.

\footnotetext{
${ }^{8}$ The complete relation between the potentials (3.29) and (2.22) is $C_{3}=-\frac{L}{\operatorname{Im} \tau} \operatorname{Im}\left(\mathrm{d} \bar{z} \wedge \mathcal{B}_{2}^{\prime}\right)$, with $\mathcal{B}_{2}^{\prime}=$ $\mathcal{B}_{2}-\frac{1}{2} \mathrm{~d} b^{1,0}, C_{3}^{(0)}=-\frac{L}{\operatorname{Im} \tau} \operatorname{Im}\left(\mathrm{d} \bar{z} \wedge \mathcal{B}_{2}^{(0)}\right)$ and $\lambda_{\alpha}=\frac{\mathrm{i}}{2 \operatorname{Im} \tau} \mathrm{d} \bar{z} \wedge \chi_{\alpha}$. Furthermore, if $\omega_{a}$ is Poincaré dual to a vertical divisor, then $\mathcal{T}_{a \alpha \beta}=-\frac{1}{2 \operatorname{Im} \tau} \mathcal{I}_{a \alpha \beta}$.
} 


\section{Conclusions}

In this paper we have studied the effective theory of type IIB/M-theory warped flux compactifications, completing and extending to the M-theory case the results of [1]. The type IIB case is discussed in section 2 while the M-theory case is considered in section 3 . The results are very similar and can be indeed related by duality.

In our discussion, a key role is played by the hidden dependence of the $G_{3} / G_{4}$ fluxes on the Kähler moduli. Such a dependence does not directly affect the simple formulas (2.13)/(3.11) for the Kähler potential. Rather, it can be taken into account just by including an additional $G_{3} / G_{4}$ dependent term — given by the last line of equations $(2.29) /(3.34)$ - in the definition of the chiral coordinates $\rho_{a}$ parametrising the Kähler moduli. This produces a manifestly $G_{3} / G_{4}$ dependent contribution to the effective Lagrangian $(2.41) /(3.40)$. More precisely, this contribution appears in the second term of the inverse kinetic matrix $(2.45) /(3.44)$ and is always positive, hence having a 'suppression effect' on the kinetic matrix $\mathcal{G}^{a b}$ of the chiral fields $\rho_{a}$. Our results reproduce, complete and supersymmetrise the bosonic effective action obtained in $[22,25]$ by dimensional reduction of IIB compactifications at fixed non-universal Kähler moduli.

There are several aspects that remain to be explored, including the following ones:

- Warping and fluxes apparently 'break' the cohomological nature of the effective theories obtained in the constant warping approximation. In particular, for the moment, the evaluation of the explicit form of the effective theory seems to require a case by case study. It would be very useful to develop efficient, model-independent topological/cohomological/algebraic-geometrical techniques to compute the explicit structure of our effective theories. This would allow for a better understanding of the physical and phenomenological implications of our results, both at a qualitative and a quantitative level.

- It would be important to further study the applications of our results to the phenomenologically more relevant framework of F-theory flux compactifications, by combining both the type IIB and M-theory perspectives, as for instance done in $[2,38]$ in the constant warping approximation. The present paper and [1] assume dynamically frozen axion-dilaton and complex structure and focus on the remaining moduli sector, but it would be clearly worthwhile to incorporate gauge and charged matter sectors into the effective theory, as well as possible dynamical complex structure moduli which may be present in the low-energy spectrum.

- In deriving our effective theories we have used the standard two-derivative type IIB/M-theory supergravity. It would be interesting to understand how to incorporate higher-derivative corrections, for instance combining our results with those of the papers $[23,24]$, in which this problem has been investigated for weakly warped M-theory compactifications. Higher derivative corrections can play an important role in phenomenological models - see for instance the reviews $[2,4]$ - and so it would be desirable to better understand how to combine them with the effects of warping, fluxes and branes. 
- Even though our effective theories have a natural applicability to string phenomenology, they may also be useful in the somewhat different context of the AdS/CFT correspondence. Indeed, as discussed in [46], by taking a rigid/decompactification limit thereof, one can obtain the 'holographic effective field theory' of strongly coupled quantum field theories. Ref. [46] considered type IIB holographic models in which the non-compact internal space is an asymptotically conical Calabi-Yau threefold and the warping is only due to mobile D3-branes, but the same logic can be applied to more general type IIB/M-theory holographic models for which the results of the present paper can be relevant.

\section{Acknowledgments}

I would like to thank Andrew Frey for correspondence and Dmitri Sorokin for comments on the manuscript. This work was partially supported by the Padua University Project CPDA144437.

\section{A IIB-theory effective theory: some details}

In this appendix we collect some technical details regarding the derivation of the effective theory of the IIB compactifications considered in section 2.

\section{A.1 Explicit form of the warped divisor volume}

In [1] it was shown how, given a divisor $D \simeq n^{a} D_{a}$, one can express the integral

$$
\frac{1}{2} \int_{D} e^{-4 A} J \wedge J
$$

in terms of the background moduli. Let us briefly review this result and its derivation.

First one can use (2.5) and (2.8) to write (A.1) as follows

$$
\frac{1}{2} a n^{a} \mathcal{I}_{a b c} v^{b} v^{c}+\frac{1}{2} \int_{X} e^{-4 A_{0}} J \wedge J \wedge \delta^{2}(D)
$$

where $\delta^{2}(D)$ is a delta-like 2-form localised on the divisor $D$. This can be written as $\delta^{2}(D)=\frac{\mathrm{i}}{2 \pi} \partial \bar{\partial} \log \left|\zeta_{D}\right|^{2}$, with $\zeta_{D}$ being a section of the line bundle $\mathcal{O}(D)$ that vanishes on $D$, so that

$$
J\lrcorner \delta^{2}(D)=-\frac{1}{4 \pi} \Delta \log \left|\zeta_{D}\right|^{2} .
$$

Furthermore, since $\omega_{a}=\mathrm{i} \partial \bar{\partial} \kappa_{a}$ is harmonic, the scalar

$$
\left.d_{a} \equiv J\right\lrcorner \omega_{a}=-\frac{1}{2} \Delta \kappa_{a}
$$

is harmonic and hence constant along $X$. We can then use the identity

$$
J\lrcorner \delta^{2}(D)=\frac{1}{4 \pi} \Delta\left(2 \pi n^{a} \kappa_{a}-\log \left|\zeta_{D}\right|^{2}\right)+n^{a} d_{a} .
$$


and (2.6) to rewrite the second term of (A.2) as

$$
\left.\int_{X} e^{-4 A_{0}}[J\lrcorner \delta^{2}(D)\right] \mathrm{dvol}_{X}=\frac{1}{4 \pi} \int_{X} e^{-4 A_{0}} \Delta\left(2 \pi n^{a} \kappa_{a}-\log \left|\zeta_{D}\right|^{2}\right) \operatorname{dvol}_{X} .
$$

Now, the key point is that the local potential $n^{a} \kappa_{a}$ defines a metric $e^{-2 \pi n^{a} \kappa_{a}}$ on the line bundle $\mathcal{O}(D)$ and then the combination $2 \pi n^{a} \kappa_{a}-\log \left|\zeta_{D}\right|^{2}$ is globally defined. This implies that we can integrate the right-hand side of (A.6) by parts and use (2.2) to get the identity

$$
\left.\int_{X} e^{-4 A_{0}}[J\lrcorner \delta^{2}(D)\right] \mathrm{dvol}_{X}=\frac{1}{\ell_{\mathrm{s}}^{4}} \int_{D}\left(2 \pi n^{a} \kappa_{a}-\log \left|\zeta_{D}\right|^{2}\right) Q_{6} .
$$

By using (2.3), we then conclude that (A.1) with $D \equiv D_{a}$ (hence $n^{b}=\delta_{a}^{b}$ ) can be rewritten as

$$
\frac{1}{2} a \mathcal{I}_{a b c} v^{b} v^{c}+\frac{1}{2} \sum_{I} \kappa_{a}\left(Z_{I}, \bar{Z}_{I} ; v\right)+h_{a}(v)-\sum_{I}\left[\log \zeta_{a}\left(Z_{I}\right)+\log \bar{\zeta}_{a}\left(\bar{Z}_{I}\right)\right]
$$

where $h_{a}(v)$ is defined in (2.32). The last term in $(\mathrm{A} .8)$ is $(\mathrm{hol}+\overline{\mathrm{hol}})$ and then, by using this formula in (2.25) and (2.26), we can discard it and identify the first three terms appearing on the right-hand side of (2.29).

\section{A.2 Derivation of kinetic terms for IIB compactifications}

In this appendix we discuss the derivation of the effective Lagrangian (2.41) from (2.13) and (2.29).

We start from some useful formulas for the implicit derivatives of the moduli. Consider the tautological identities $\frac{\partial \operatorname{Re} \rho_{a}}{\partial \operatorname{Re} \rho_{b}}=\delta_{a}^{b}, \frac{\partial \operatorname{Re} \rho_{a}}{\partial Z_{I}^{i}}=0$ and $\frac{\partial \operatorname{Re} \rho_{a}}{\partial \beta^{\alpha}}=0$. By using (2.36) we can rewrite them as follows

$$
\begin{aligned}
\frac{1}{2} \mathcal{I}_{a c d} v^{c} v^{d} \frac{\partial a}{\partial \operatorname{Re} \rho_{b}}+\mathcal{M}_{a c} \frac{\partial v^{c}}{\partial \operatorname{Re} \rho_{b}} & =\delta_{a}^{b} \\
\frac{1}{2} \mathcal{I}_{a b c} v^{b} v^{c} \frac{\partial a}{\partial Z_{I}^{i}}+\mathcal{M}_{a b} \frac{\partial v^{b}}{\partial Z_{I}^{i}}+\frac{1}{2} \mathcal{A}_{a i}^{I} & =0 \\
\frac{1}{2} \mathcal{I}_{a b c} v^{b} v^{c} \frac{\partial a}{\partial \beta^{\alpha}}+\mathcal{M}_{a b} \frac{\partial v^{b}}{\partial \beta^{\alpha}}+\frac{\mathrm{i}}{2 \operatorname{Im} \tau} \mathcal{I}_{a \alpha \beta} \operatorname{Im} \beta^{\beta} & =0
\end{aligned}
$$

where $\mathcal{A}_{a i}^{I}$ is defined in (2.44). By contracting (A.9) with $v^{a}$ and using (2.39) together with (2.9) and its corollary $\mathcal{I}_{a b c} v^{a} v^{b} \frac{\partial v^{c}}{\partial \operatorname{Re} \rho^{d}}=0$, we get

$$
\frac{\partial a}{\partial \operatorname{Re} \rho_{a}}=\frac{1}{3 \mathrm{v}_{0}} v^{a}, \quad \frac{\partial a}{\partial Z_{I}^{i}}=-\frac{1}{6 \mathrm{v}_{0}} v^{a} \mathcal{A}_{a i}^{I}, \quad \frac{\partial a}{\partial \beta^{\alpha}}=-\frac{\mathrm{i}}{6 \mathrm{v}_{0} \operatorname{Im} \tau} v^{a} \mathcal{I}_{a \alpha \beta} \operatorname{Im} \beta^{\beta} .
$$

Using these relations back in (A.9) and taking again (2.39) into account, one also gets

$$
\begin{aligned}
\frac{\partial v^{a}}{\partial \operatorname{Re} \rho_{b}} & =\mathcal{M}^{a b}-\frac{1}{6 \mathrm{v}_{0} a} v^{a} v^{b} \\
\frac{\partial v^{a}}{\partial Z_{I}^{i}} & =-\frac{1}{2}\left(\mathcal{M}^{a b}-\frac{1}{6 \mathrm{v}_{0} a} v^{a} v^{b}\right) \mathcal{A}_{b i}^{I} \\
\frac{\partial v^{a}}{\partial \beta^{\alpha}} & =-\frac{\mathrm{i}}{2 \operatorname{Im} \tau}\left(\mathcal{M}^{a b}-\frac{1}{6 \mathrm{v}_{0} a} v^{a} v^{b}\right) \mathcal{I}_{b \alpha \beta} \operatorname{Im} \beta^{\beta}
\end{aligned}
$$

where $\mathcal{M}^{a b}$ is the inverse of $\mathcal{M}_{a b}$. 
Consider now the Kähler potential (2.13). By taking into account that it does not depend on $\operatorname{Im} \rho_{a}$ and $\operatorname{Re} \beta^{\alpha}$ and and using (A.10) we can compute

$$
\frac{\partial K}{\partial \rho_{a}}=-\frac{v^{a}}{2 \mathrm{v}_{0} a}, \quad \frac{\partial K}{\partial Z_{I}^{i}}=\frac{1}{2 \mathrm{v}_{0} a} v^{a} \mathcal{A}_{a i}^{I}, \quad \frac{\partial K}{\partial \beta^{\alpha}}=\frac{\mathrm{i}}{2 \mathrm{v}_{0} a \operatorname{Im} \tau} v^{a} \mathcal{I}_{a \alpha \beta} \operatorname{Im} \beta^{\beta} .
$$

Then, by using again (A.10) together with (A.11) and (2.34), we obtain the second derivatives of $K$ :

$$
\begin{aligned}
\frac{\partial^{2} K}{\partial \rho_{a} \partial \bar{\rho}_{b}} & =-\frac{1}{4 \mathrm{v}_{0} a}\left(\mathcal{M}^{a b}-\frac{1}{2 \mathrm{v}_{0} a} v^{a} v^{b}\right) \equiv \mathcal{G}^{a b} \\
\frac{\partial^{2} K}{\partial \rho_{a} \partial \bar{Z}_{I}^{\bar{l}}} & =-\mathcal{G}^{a b} \overline{\mathcal{A}}_{b \bar{\imath}}^{I} \\
\frac{\partial^{2} K}{\partial Z_{I}^{i} \partial \bar{Z}_{J}^{\bar{\jmath}}} & =\mathcal{G}^{a b} \mathcal{A}_{a i}^{I} \overline{\mathcal{A}}_{b \bar{\jmath}}^{J}+\frac{1}{2 \mathrm{v}_{0} a} g_{i \bar{\jmath}}\left(Z_{I}, \bar{Z}_{I}\right) \\
\frac{\partial^{2} K}{\partial \rho_{a} \partial \bar{\beta}^{\alpha}} & =\frac{\mathrm{i}}{\operatorname{Im} \tau} \mathcal{G}^{a b} \mathcal{I}_{b \alpha \beta} \operatorname{Im} \beta^{\beta} \\
\frac{\partial^{2} K}{\partial \beta^{\alpha} \partial \bar{Z}_{I}^{\bar{\imath}}} & =\frac{\mathrm{i}}{\operatorname{Im} \tau} \mathcal{G}^{a b} \overline{\mathcal{A}}_{a \bar{\imath}}^{I} \mathcal{I}_{b \alpha \beta} \operatorname{Im} \beta^{\beta}, \\
\frac{\partial^{2} K}{\partial \beta^{\alpha} \partial \bar{\beta}^{\beta}} & =-\frac{1}{4 \mathrm{v}_{0} a \operatorname{Im} \tau} v^{a} \mathcal{I}_{a \alpha \beta}+\frac{1}{(\operatorname{Im} \tau)^{2}} \mathcal{G}^{a b} \mathcal{I}_{a \alpha \gamma} \mathcal{I}_{b \beta \delta} \operatorname{Im} \beta^{\gamma} \operatorname{Im} \beta^{\delta}
\end{aligned}
$$

These formulas can be used to compute the effective theory. In particular, the bosonic effective Lagrangian takes the form (2.41).

\section{B M-theory effective theory: some details}

In this appendix we derive some formulas presented in section 3.

\section{B.1 Warped divisor volumes in M-theory}

We want to make more explicit the dependence of (3.30) on the background moduli, following [1]. First we can use (3.6) and the homological decompositions $[S]=m^{A}\left[D_{A}\right]$ to rewrite (3.30) as follows:

$$
\left.\frac{1}{3 !} \int_{S} e^{-6 D} J \wedge J \wedge J=\frac{1}{3 !} c m^{A} \mathcal{I}_{A B C D} v^{B} v^{C} v^{D}+\int_{X} e^{-6 D_{0}}[J\lrcorner \delta^{2}(S)\right] \mathrm{d} v o l_{Y} .
$$

One can now use the identity $\delta^{2}(S)=\frac{1}{2 \pi} \mathrm{i} \partial \bar{\partial} \log \left|\zeta_{S}\right|^{2}$ to deduce that

$$
J\lrcorner \delta^{2}(S)=-\frac{1}{4 \pi} \Delta \log \left|\zeta_{S}\right|^{2}
$$

where $\zeta_{S}$ is a section of the line bundle $\mathcal{O}(S)$ that vanishes on $S$. Furthermore, since $\omega_{A}=\mathrm{i} \partial \bar{\partial} \kappa_{A}$ is harmonic, we can observe that

$$
J\lrcorner \omega_{A}=-\frac{1}{2} \Delta \kappa_{A}
$$


is a constant. Then, recalling (3.7) and (3.4), we can combine (B.2) and (B.3) to obtain the identity

$$
\left.\int_{X} e^{-6 D_{0}}[J\lrcorner \delta^{2}(S)\right] \mathrm{dvol}_{Y}=\frac{1}{4 \pi \ell_{\mathrm{M}}^{6}} \int_{Y}\left(2 \pi m^{A} \kappa_{A}-\log \left|\zeta_{S}\right|^{2}\right) Q_{6}
$$

where $2 \pi m^{A} \kappa_{A}-\log \left|\zeta_{S}\right|^{2}$ is a globally defined function. Hence, by using (3.5), (B.1) finally takes the form

$$
\begin{aligned}
\frac{1}{3 !} \int_{S} e^{-6 D} J \wedge J \wedge J= & \frac{1}{3 !} c m^{A} \mathcal{I}_{A B C D} v^{B} v^{C} v^{D}+\frac{1}{2} \sum_{I} m^{A} \kappa_{A}\left(Z_{I}, \bar{Z}_{I} ; v\right) \\
& +\frac{1}{4 \pi \ell_{\mathrm{M}}^{6}} \int_{Y}\left(2 \pi m^{A} \kappa_{A}-\log \left|\zeta_{S}\right|^{2}\right)\left(\frac{1}{2} G_{4} \wedge G_{4}-\ell_{\mathrm{M}}^{6} I_{8}\right) \\
& -\frac{1}{4 \pi} \sum_{I}\left[\log \zeta_{S}\left(Z_{I}\right)+\log \bar{\zeta}_{S}\left(\bar{Z}_{I}\right)\right]
\end{aligned}
$$

By choosing $S \rightarrow S_{a}$ and $m^{A} \rightarrow m_{a}^{A}$ and discarding the (hol $+\overline{\text { hol }}$ ) term appearing in the last line, we obtain (3.32).

\section{B.2 Variation of $\operatorname{Re} \rho_{a}$ in M-theory}

We want to compute how $\operatorname{Re} \rho_{a}$ varies under a deformation $\delta v^{a}$ of the non-universal Kähler moduli $\delta v^{a}$ preserving the constraint (3.14) and the cohomological primitivity condition (3.17).

We first compute the variation of the potentials $\kappa_{A}(z, \bar{z} ; v)$, starting from $\delta \omega_{A}=$ $\mathrm{i} \partial \bar{\partial} \delta \kappa_{A}$, following [1]. This can be done without restricting to deformations preser-

ving (3.17). Hence, for the moment, we consider more general variation $\delta u^{A}$ preserving (3.14). Defining

$$
\left.d_{A} \equiv J\right\lrcorner \omega_{A}
$$

(which is a constant along $Y$ ) one can check that we can write

$$
\left.\Delta \delta \kappa_{A}=-2\left(\delta d_{A}+\delta u^{B} \omega_{B}\right\lrcorner \omega_{A}\right) .
$$

Noticing that

$$
\left.\delta d_{A}=\frac{1}{2 \mathrm{w}_{0}} \mathcal{I}_{A B C D} u^{C} u^{D} \delta u^{B}=-\frac{1}{\mathrm{w}_{0}} \delta u^{B} \int_{Y} \omega_{B}\right\lrcorner \omega_{A} \mathrm{dvol}_{Y}
$$

we see that (B.7) is indeed integrable. The solution can be written as

$$
\delta \kappa_{A}(y ; v)=\delta u^{B} \int_{Y, y^{\prime}} G\left(y, y^{\prime}\right)\left(J \wedge J \wedge \omega_{A} \wedge \omega_{B}\right)\left(y^{\prime}\right)
$$

where $G\left(y, y^{\prime}\right)=G\left(y^{\prime}, y\right)$ is the Green's function associated with the Kähler metric $\mathrm{d} s_{Y}^{2}$.

Restricting back to variations $\delta v^{a}$ preserving the primitivity condition (3.17), we can now write

$$
\delta \kappa_{A}(y ; v)=\delta v^{a} \int_{Y, y^{\prime}} G\left(y, y^{\prime}\right)\left(J \wedge J \wedge \omega_{a} \wedge \omega_{A}\right)\left(y^{\prime}\right)
$$


where $\omega_{a}=m_{a}^{A} \omega_{A}$. By writing $e^{-6 D_{0}}$ appearing in (3.6) as

$$
e^{-6 D_{0}(y)}=\frac{1}{\ell_{\mathrm{M}}^{8}} \int_{Y} G\left(y ; y^{\prime}\right) Q_{8}
$$

it is not difficult to check that the variation (B.10) in (3.34), combined with the variation of $\frac{1}{3 !} c \mathcal{I}_{a A B C} u^{A} u^{C} u^{D}$, produces

$$
\frac{1}{2} \delta v^{b} \int_{Y} e^{-6 D} J \wedge J \wedge \omega_{a} \wedge \omega_{b} \quad \subset \quad \delta \rho_{a} .
$$

On the other hand, in computing $\delta \rho_{a}$ we also need to take into account the hidden $G_{4}$ dependence on the $v^{a}$ Kähler moduli discussed in subsection 3.3. By using (3.20) and (3.28) it is straightforward to compute the corresponding variation in (3.34). In particular the variation of $G_{4}$ in $h(v)$ combines with the variation of the last term (the $\int_{S_{a}}$ integral), producing

$$
\frac{1}{2 \ell_{\mathrm{M}}^{6}} \delta v^{b} \int_{Y} \omega_{a} \wedge \Lambda_{b}^{1,1} \wedge G_{4} \quad \subset \quad \delta \rho_{a} .
$$

By combining (B.12) and (B.13) one obtains (3.36).

\section{B.3 Useful formulas for M-theory effective theory}

Starting from the definition (3.34) and using (3.36), one can rewrite the tautological identities $\frac{\partial \operatorname{Re} \rho_{a}}{\partial \operatorname{Re} \rho_{b}}=\delta_{a}^{b}, \frac{\partial \operatorname{Re} \rho_{a}}{\partial Z_{I}^{i}}=0$ and $\frac{\partial \operatorname{Re} \rho_{a}}{\partial \beta^{\alpha}}=0$ as follows

$$
\begin{array}{r}
\frac{1}{3 !} \mathcal{I}_{a A B C} u^{A} u^{B} u^{C} \frac{\partial c}{\partial \operatorname{Re} \rho_{b}}+\mathcal{N}_{a c} \frac{\partial v^{c}}{\partial \operatorname{Re} \rho_{b}}=\delta_{a}^{b} \\
\frac{1}{3 !} \mathcal{I}_{a A B C} u^{A} u^{B} u^{C} \frac{\partial c}{\partial Z_{I}^{i}}+\mathcal{N}_{a b} \frac{\partial v^{b}}{\partial Z_{I}^{i}}+\frac{1}{2} \mathcal{A}_{a i}^{I}=0 \\
\frac{1}{3 !} \mathcal{I}_{a A B C} u^{A} u^{B} u^{C} \frac{\partial c}{\partial \beta^{\alpha}}+\mathcal{N}_{a b} \frac{\partial v^{b}}{\partial \beta^{\alpha}}+\frac{1}{2} \mathcal{T}_{a \alpha \bar{\beta}} \bar{\beta}^{\bar{\beta}}=0
\end{array}
$$

where $\mathcal{A}_{a i}^{I}$ is defined in (3.42). By contracting (B.14) with $v^{a}$ and recalling (3.39), we get

$$
\frac{\partial c}{\partial \operatorname{Re} \rho_{a}}=\frac{1}{4 \mathrm{w}_{0}} v^{a}, \quad \frac{\partial c}{\partial Z_{I}^{i}}=-\frac{1}{8 \mathrm{w}_{0}} v^{a} \mathcal{A}_{a i}^{I}, \quad \frac{\partial c}{\partial \beta^{\alpha}}=-\frac{1}{8 \mathrm{w}_{0}} v^{a} \mathcal{T}_{a \alpha} \bar{\beta} \bar{\beta}^{\bar{\beta}}
$$

By using these relations, (B.14) also give

$$
\begin{aligned}
\frac{\partial v^{a}}{\partial \operatorname{Re} \rho_{b}} & =\mathcal{N}^{a b}-\frac{1}{12 \mathrm{w}_{0} c} v^{a} v^{b} \\
\frac{\partial v^{a}}{\partial Z_{I}^{i}} & =-\frac{1}{2}\left(\mathcal{N}^{a b}-\frac{1}{12 \mathrm{w}_{0} c} v^{a} v^{b}\right) \mathcal{A}_{b i}^{I} \\
\frac{\partial v^{a}}{\partial \beta^{\alpha}} & =-\frac{1}{2}\left(\mathcal{N}^{a b}-\frac{1}{12 \mathrm{w}_{0} c} v^{a} v^{b}\right) \mathcal{T}_{b \alpha \bar{\beta}} \bar{\beta}^{\bar{\beta}}
\end{aligned}
$$

where $\mathcal{N}^{a b}$ is the inverse of $\mathcal{N}_{a b}$. 
Open Access. This article is distributed under the terms of the Creative Commons Attribution License (CC-BY 4.0), which permits any use, distribution and reproduction in any medium, provided the original author(s) and source are credited.

\section{References}

[1] L. Martucci, Warping the Kähler potential of F-theory/IIB flux compactifications, JHEP 03 (2015) 067 [arXiv: 1411.2623] [INSPIRE].

[2] F. Denef, Les Houches Lectures on Constructing String Vacua, arXiv:0803.1194 [INSPIRE].

[3] T. Weigand, Lectures on F-theory compactifications and model building, Class. Quant. Grav. 27 (2010) 214004 [arXiv: 1009.3497] [InSPIRE].

[4] A. Maharana and E. Palti, Models of Particle Physics from Type IIB String Theory and F-theory: A Review, Int. J. Mod. Phys. A 28 (2013) 1330005 [arXiv:1212.0555] [InSPIRE].

[5] P. Koerber and L. Martucci, From ten to four and back again: How to generalize the geometry, JHEP 08 (2007) 059 [arXiv:0707.1038] [INSPIRE].

[6] L. Martucci, On moduli and effective theory of $N=1$ warped flux compactifications, JHEP 05 (2009) 027 [arXiv: 0902.4031] [INSPIRE].

[7] M. Graña and J. Polchinski, Supersymmetric three form flux perturbations on AdS $S_{5}$, Phys. Rev. D 63 (2001) 026001 [hep-th/0009211] [INSPIRE].

[8] S.S. Gubser, Supersymmetry and F-theory realization of the deformed conifold with three form flux, hep-th/0010010 [INSPIRE].

[9] S.B. Giddings, S. Kachru and J. Polchinski, Hierarchies from fluxes in string compactifications, Phys. Rev. D 66 (2002) 106006 [hep-th/0105097] [INSPIRE].

[10] K. Dasgupta, G. Rajesh and S. Sethi, M theory, orientifolds and G-flux, JHEP 08 (1999) 023 [hep-th/9908088] [INSPIRE].

[11] O. DeWolfe and S.B. Giddings, Scales and hierarchies in warped compactifications and brane worlds, Phys. Rev. D 67 (2003) 066008 [hep-th/0208123] [INSPIRE].

[12] S.B. Giddings and A. Maharana, Dynamics of warped compactifications and the shape of the warped landscape, Phys. Rev. D 73 (2006) 126003 [hep-th/0507158] [INSPIRE].

[13] C.P. Burgess et al., Warped Supersymmetry Breaking, JHEP 04 (2008) 053 [hep-th/0610255] [INSPIRE].

[14] G. Shiu, G. Torroba, B. Underwood and M.R. Douglas, Dynamics of Warped Flux Compactifications, JHEP 06 (2008) 024 [arXiv: 0803.3068] [INSPIRE].

[15] M.R. Douglas and G. Torroba, Kinetic terms in warped compactifications, JHEP 05 (2009) 013 [arXiv: 0805.3700] [INSPIRE].

[16] A.R. Frey, G. Torroba, B. Underwood and M.R. Douglas, The Universal Kähler Modulus in Warped Compactifications, JHEP 01 (2009) 036 [arXiv: 0810.5768] [INSPIRE].

[17] F. Marchesano, P. McGuirk and G. Shiu, Open String Wavefunctions in Warped Compactifications, JHEP 04 (2009) 095 [arXiv: 0812.2247] [INSPIRE].

[18] H.-Y. Chen, Y. Nakayama and G. Shiu, On D3-brane Dynamics at Strong Warping, Int. J. Mod. Phys. A 25 (2010) 2493 [arXiv:0905.4463] [InSPIRE]. 
[19] B. Underwood, A Breathing Mode for Warped Compactifications, Class. Quant. Grav. 28 (2011) 195013 [arXiv: 1009.4200] [INSPIRE].

[20] F. Marchesano, P. McGuirk and G. Shiu, Chiral matter wavefunctions in warped compactifications, JHEP 05 (2011) 090 [arXiv: 1012.2759] [INSPIRE].

[21] T.W. Grimm, D. Klevers and M. Poretschkin, Fluxes and Warping for Gauge Couplings in F-theory, JHEP 01 (2013) 023 [arXiv: 1202.0285] [INSPIRE].

[22] A.R. Frey and J. Roberts, The Dimensional Reduction and Kähler Metric of Forms In Flux and Warping, JHEP 10 (2013) 021 [arXiv:1308.0323] [INSPIRE].

[23] T.W. Grimm, T.G. Pugh and M. Weissenbacher, The effective action of warped M-theory reductions with higher derivative terms — part I, JHEP 01 (2016) 142 [arXiv:1412.5073] [INSPIRE].

[24] T.W. Grimm, T.G. Pugh and M. Weissenbacher, The effective action of warped M-theory reductions with higher-derivative terms - Part II, JHEP 12 (2015) 117 [arXiv:1507.00343] [INSPIRE].

[25] B. Cownden, A.R. Frey, M.C.D. Marsh and B. Underwood, Dimensional Reduction for D3-brane Moduli, JHEP 12 (2016) 139 [arXiv:1609.05904] [INSPIRE].

[26] E. Cremmer, S. Ferrara, C. Kounnas and D.V. Nanopoulos, Naturally Vanishing Cosmological Constant in $N=1$ Supergravity, Phys. Lett. B 133 (1983) 61 [InSPIRE].

[27] J.R. Ellis, A.B. Lahanas, D.V. Nanopoulos and K. Tamvakis, No-Scale Supersymmetric Standard Model, Phys. Lett. B 134 (1984) 429 [INSPIRE].

[28] C. Vafa, Evidence for F-theory, Nucl. Phys. B 469 (1996) 403 [hep-th/9602022] [INSPIRE].

[29] K. Becker and M. Becker, M theory on eight manifolds, Nucl. Phys. B 477 (1996) 155 [hep-th/9605053] [INSPIRE].

[30] K. Becker and M. Becker, Supersymmetry breaking, M-theory and fluxes, JHEP 07 (2001) 038 [hep-th/0107044] [INSPIRE].

[31] D.Z. Freedman and A Van Proeyen, Supergravity, Cambridge University Press (2012).

[32] L. Martucci and P. Smyth, Supersymmetric D-branes and calibrations on general $N=1$ backgrounds, JHEP 11 (2005) 048 [hep-th/0507099] [INSPIRE].

[33] T.W. Grimm and J. Louis, The effective action of $N=1$ Calabi-Yau orientifolds, Nucl. Phys. B 699 (2004) 387 [hep-th/0403067] [INSPIRE].

[34] P. Binetruy, G. Girardi and R. Grimm, Supergravity couplings: A geometric formulation, Phys. Rept. 343 (2001) 255 [hep-th/0005225] [INSPIRE].

[35] T.W. Grimm, The effective action of type-II Calabi-Yau orientifolds, Fortsch. Phys. 53 (2005) 1179 [hep-th/0507153] [INSPIRE].

[36] M. Haack and J. Louis, Duality in heterotic vacua with four supercharges, Nucl. Phys. B $\mathbf{5 7 5}$ (2000) 107 [hep-th/9912181] [INSPIRE].

[37] M. Haack and J. Louis, M theory compactified on Calabi-Yau fourfolds with background flux, Phys. Lett. B 507 (2001) 296 [hep-th/0103068] [INSPIRE].

[38] T.W. Grimm, The $N=1$ effective action of F-theory compactifications, Nucl. Phys. B $\mathbf{8 4 5}$ (2011) 48 [arXiv: 1008.4133] [INSPIRE]. 
[39] M.J. Duff, J.T. Liu and R. Minasian, Eleven-dimensional origin of string-string duality: A one loop test, Nucl. Phys. B 452 (1995) 261 [hep-th/9506126] [INSPIRE].

[40] S.M. Kuzenko, U. Lindström, M. Roček, I. Sachs and G. Tartaglino-Mazzucchelli, Three-dimensional $\mathcal{N}=2$ supergravity theories: From superspace to components, Phys. Rev. D 89 (2014) 085028 [arXiv:1312.4267] [INSPIRE].

[41] E. Witten, On flux quantization in M-theory and the effective action, J. Geom. Phys. 22 (1997) 1 [hep-th/9609122] [INSPIRE].

[42] E. Witten, Five-brane effective action in M-theory, J. Geom. Phys. 22 (1997) 103 [hep-th/9610234] [INSPIRE].

[43] P. Pasti, D.P. Sorokin and M. Tonin, Covariant action for a $D=11$ five-brane with the chiral field, Phys. Lett. B 398 (1997) 41 [hep-th/9701037] [INSPIRE].

[44] I.A. Bandos, K. Lechner, A. Nurmagambetov, P. Pasti, D.P. Sorokin and M. Tonin, Covariant action for the superfive-brane of M-theory, Phys. Rev. Lett. 78 (1997) 4332 [hep-th/9701149] [INSPIRE].

[45] S. Gukov, C. Vafa and E. Witten, CFT's from Calabi-Yau four folds, Nucl. Phys. B 584 (2000) 69 [Erratum ibid. B 608 (2001) 477] [hep-th/9906070] [INSPIRE].

[46] L. Martucci and A. Zaffaroni, Holographic Effective Field Theories, JHEP 06 (2016) 166 [arXiv:1603.04470] [INSPIRE]. 